\title{
Surgical Management of Peri-implantitis
}

\author{
Ausra Ramanauskaite $^{1} \cdot$ Karina Obreja $^{1}$ • Frank Schwarz ${ }^{1}$ \\ Published online: 1 August 2020 \\ (C) The Author(s) 2020
}

\begin{abstract}
Purpose of Review To provide an overview of current surgical peri-implantitis treatment options.

Recent Findings Surgical procedures for peri-implantitis treatment include two main approaches: non-augmentative and augmentative therapy. Open flap debridement (OFD) and resective treatment are non-augmentative techniques that are indicated in the presence of horizontal bone loss in aesthetically nondemanding areas. Implantoplasty performed adjunctively at supracrestally and buccally exposed rough implant surfaces has been shown to efficiently attenuate soft tissue inflammation compared to control sites. However, this was followed by more pronounced soft tissue recession. Adjunctive augmentative measures are recommended at peri-implantitis sites exhibiting intrabony defects with a minimum depth of 3 mm and in the presence of keratinized mucosa. In more advanced cases with combined defect configurations, a combination of augmentative therapy and implantoplasty at exposed rough implant surfaces beyond the bony envelope is feasible.

Summary For the time being, no particular surgical protocol or material can be considered as superior in terms of long-term periimplant tissue stability.
\end{abstract}

Keywords Peri-implantitis · Treatment · Surgical therapy

\section{Introduction}

Peri-implantitis is a plaque-associated pathological condition occurring around dental implants that results in a breakdown of the supporting tissues $[1 \bullet, 2 \bullet \bullet]$. Clinically, peri-implantitisaffected sites exhibit bleeding on probing (BOP) and/or suppuration (Supp), increased probing depths (PDs), and/or recession of the peri-implant mucosal margin in addition to radiographic bone loss compared to previous examination [1•]. Untreated disease progresses in nonlinear accelerating pattern and finally leads to a loss of the implant $[3 \bullet \bullet, 4]$. As the number of patients undergoing restorative therapy through dental implants increases, peri-implantitis is considered to be a major and growing problem in dentistry [4].

The primary goal of peri-implantitis treatment has been established as a resolution of the inflammation and a prevention

This article is part of the Topical Collection on Peri-implantitis

Ausra Ramanauskaite

Ramanauskaite@med.uni-frankfurt.de

1 Department of Oral Surgery and Implantology, Carolinum, Goethe University, Frankfurt, Germany of further bone loss [5]. To achieve these treatment endpoints, it is currently accepted that surgical approaches that allow adequate access to the contaminated implant surface are required [6-8]. Indeed, numerous peri-implantitis surgical treatment protocols have been proposed, which basically can be categorized into two main modalities: non-augmentative and augmentative.

The aim of this narrative review is to provide an overview of various peri-implantitis surgical treatment strategies with regard to their indications, performance, and efficacy.

\section{Non-augmentative Approaches}

\section{Open Flap Debridement}

In order to achieve the resolution of inflammation and arrest further bone loss, decontamination of the implant's surface is of critical importance [9]. Open flap debridement (OFD) is a surgical technique aimed at gaining access to the implant surfaces to facilitate decontamination. 


\section{Procedure}

The surgery consists of the following steps:

- Access to the lesion

- Removal of granulation tissues

- Decontamination of the implant surface

- Suturing with or without apical flap positioning

During OFD surgery, implant surface is mechanically debrided with titanium or plastic curettes or titanium brushes, with or without the adjunctive application of antimicrobials (Table 1) $[8,11,12,13,15]$. The effectiveness of different decontamination approaches has been assessed in randomized clinical investigations (RCTs) [10••, 13, 14]. Particularly, significantly greater reductions of initial PD values as well as stable bone levels were obtained at implant sites debrided with titanium brushes compared with those cleaned with plastic curettes or an air-abrasive device [13]. Repeated applications of local antibiotics (minocycline) led to superior therapeutic outcomes (i.e., greater PD reductions and increased marginal bone levels) compared with those in the placebo group [14]. In contrast to the abovementioned decontamination approaches, the additional use of a diode laser provided comparable treatment outcomes to those of mechanical debridement alone (i.e., plastic curettes and cotton pellets soaked in sterile saline) $[10 \cdot \bullet]$. It should, however, be noted that all of the abovementioned controlled clinical investigations involved a follow-up period limited to 6 months and thus do not permit an assessment of the long-term impacts of the implant surface decontamination method on the treatment outcomes following OFD. Regarding the rationale for adjunctive systemic antibiotic therapy, as demonstrated by a 1-year follow-up controlled clinical investigation, prescribing systemic antibiotics did not lead to improved therapeutic outcomes, as compared with those in the control group [12].

\section{Outcomes of the Therapy}

Peri-implantitis management with the OFD approach significantly reduced inflammation signs (BOP, PD values, and Supp) compared with the baseline situation $[8,10 \bullet$, $11,12,13]$. However, a postoperative soft tissue recession of 1.9 to $1.8 \mathrm{~mm}$ occurred after 1 year and 5 years, respectively $[8,11]$.

Treatment success, defined as PD $\leq 5 \mathrm{~mm}$, no BOP, no Supp, and no bone loss $\geq 0.5 \mathrm{~mm}$, after 6 months was achieved in $33 \%$ of the implants mechanically treated with titanium brushes, whereas lower values were noted for the sites where debridement was performed with plastic curettes or an air-abrasive device (22\% and $27 \%$, respectively; $p<0.05)$ [13]. According to the same definition, success was obtained in $47 \%$ of patients with postoperative antibiotics and $25 \%$ without systemic antibiotic prescription after 1 year; however, no significant difference was found between the groups ( $p=0.2$ ) [12]. Contrarily, disease resolution (defined as $\mathrm{PD}<5 \mathrm{~mm}$, no BOP/Supp, and no bone loss) was more frequently noted at the sites treated with locally repeated minocycline applications (intraoperatively, after 1 month, 3 months, and 6 months) compared to the controls (test $66.7 \%$, control $36.3 \%$ of the implants) [14]. Over a 5 -year follow-up, treatment success (the absence of $\mathrm{PD} \geq 5 \mathrm{~mm}$ with concomitant BOP and Supp and the absence of additional bone loss) was yielded in $63 \%$ of the patients that adhered to a regular supportive therapy [11].

\section{Resective Therapy}

Resective peri-implantitis therapy involves reducing or eliminating pathological peri-implant pockets, apical positioning of the mucosal flap, or recontouring of the bone with or without implantoplasty [16]. The indication of this surgical approach includes the presence of horizontal bone loss with exposed implant threads in non-aesthetic areas [16].

\section{Procedure}

The following steps should be followed during resective periimplantitis therapy (Fig. 1):

- Access to the defect

- Removal of inflamed tissues

- Decontamination of the implant surface

- Performance of resective therapy by means of osseous recontouring with or without implantoplasty

- Apical positioning of the mucosal flap

Mechanical debridement combined with antibacterial agent application (e.g., chlorhexidine gluconate (CHX), hydrogen peroxide, sterile saline, phosphoric acid, or antibiotic gel) is the most common method for decontaminating implant surfaces [15, 17-25] (Table 2). Clinical comparative studies have shown that the adjunctive use of phosphoric acid or CHX application with or without cetylpyridinium chloride was not superior to control methods (e.g., sterile saline alone) [17-20, 22]. Adjunctive systemic antibiotics regimens had no impact on disease resolution (i.e., $\mathrm{PD} \leq 5 \mathrm{~mm}$, no BOP/Supp, bone loss $\leq 0.5 \mathrm{~mm}$ ) in implants with nonmodified surfaces, whereas they had a positive effect on treatment success on implants with modified surfaces [20]. Over a 3-year period, however, the increased treatment success by systemic antibiotics at nonmodified surfaces was not sustained [19].

Implant surface modification, or implantoplasty, was suggested to be used as an adjunct to the resective therapy of periimplantitis [24, 25]. Implantoplasty is aimed at removing 


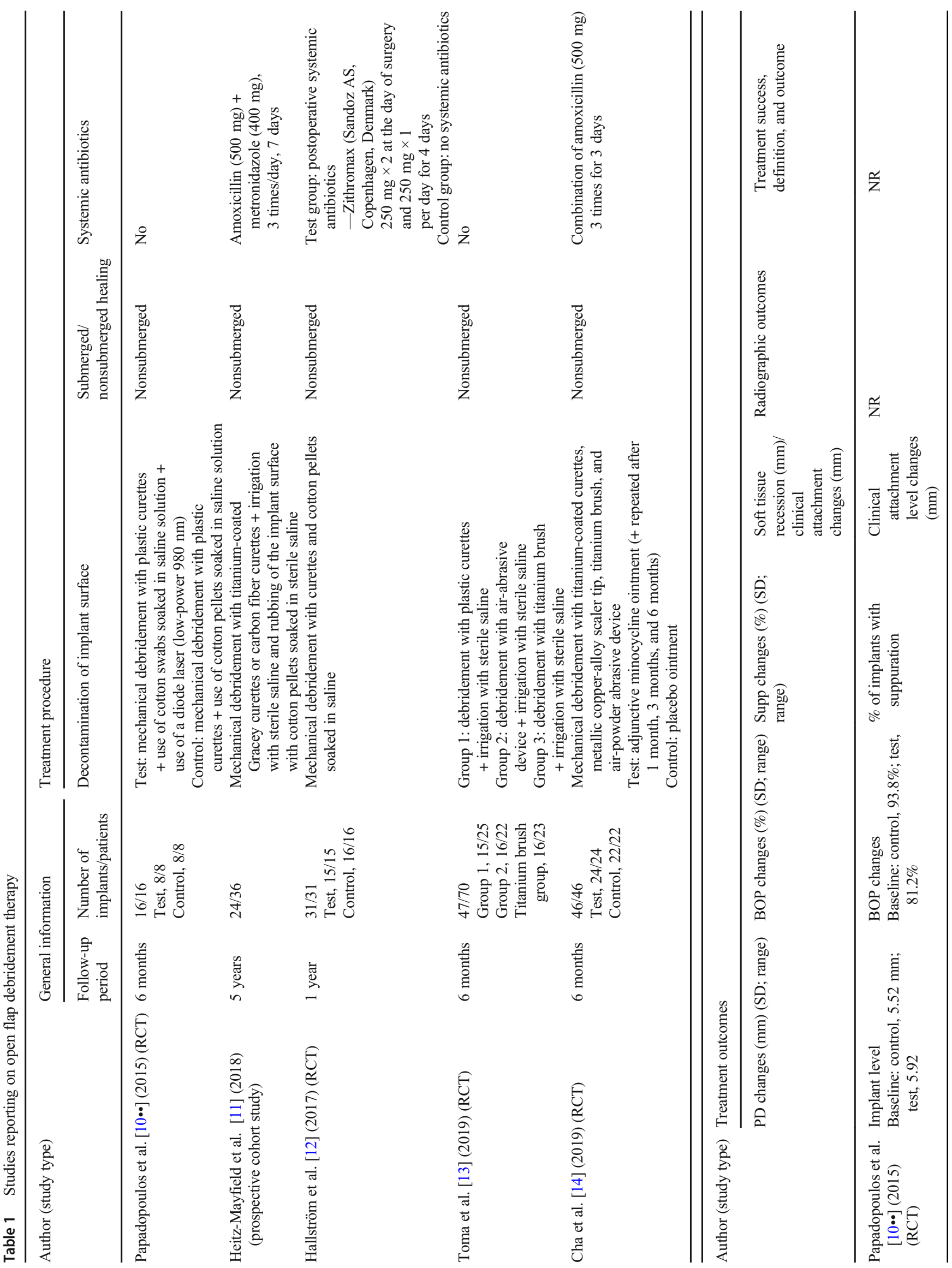




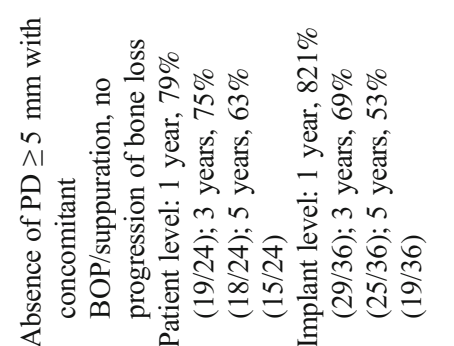

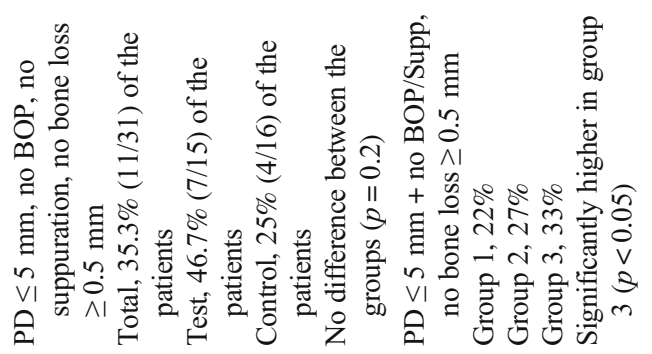
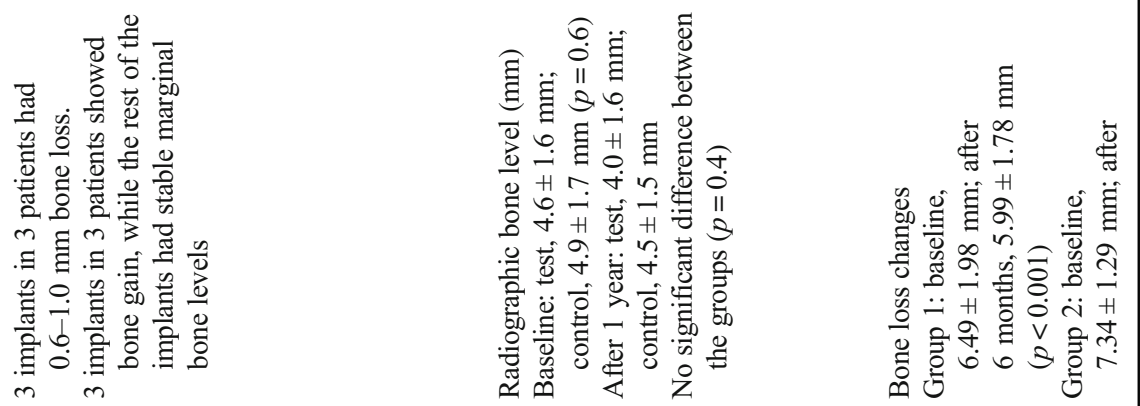

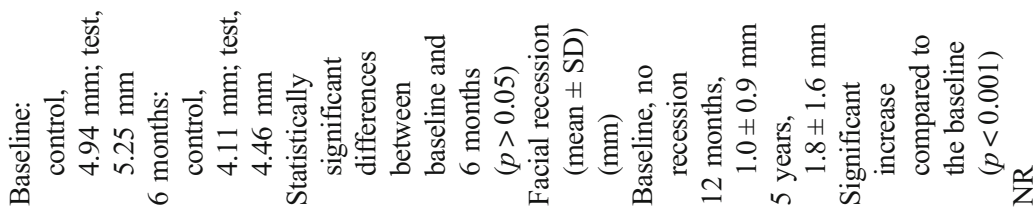

学

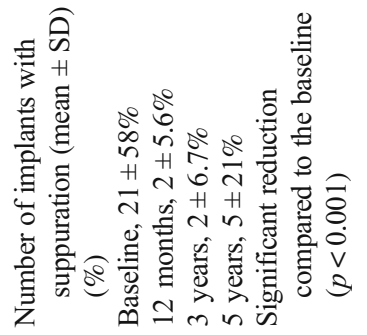

艺

弚

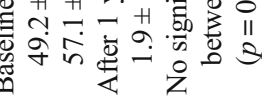

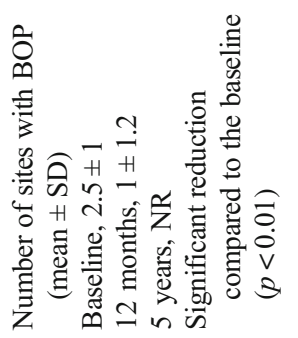

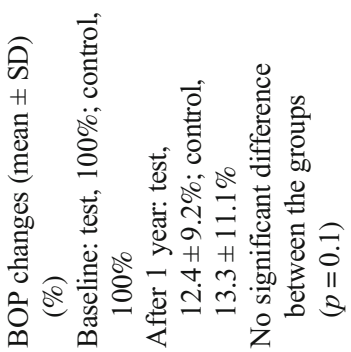

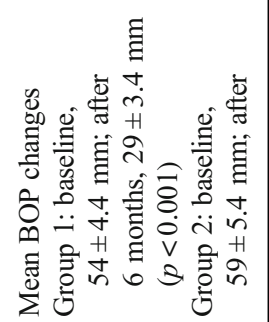
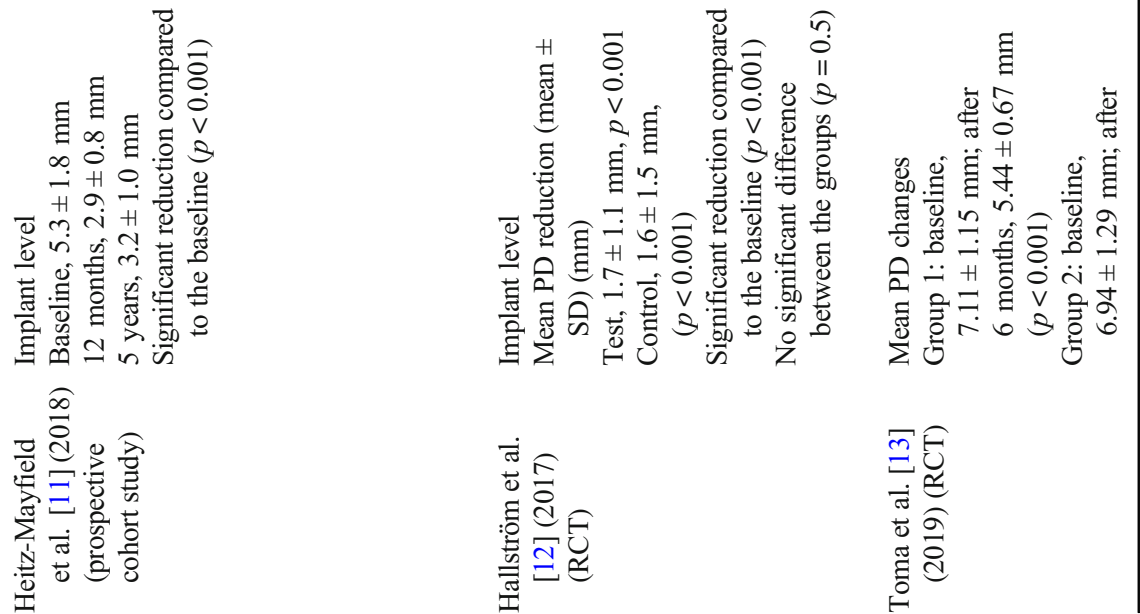

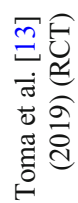




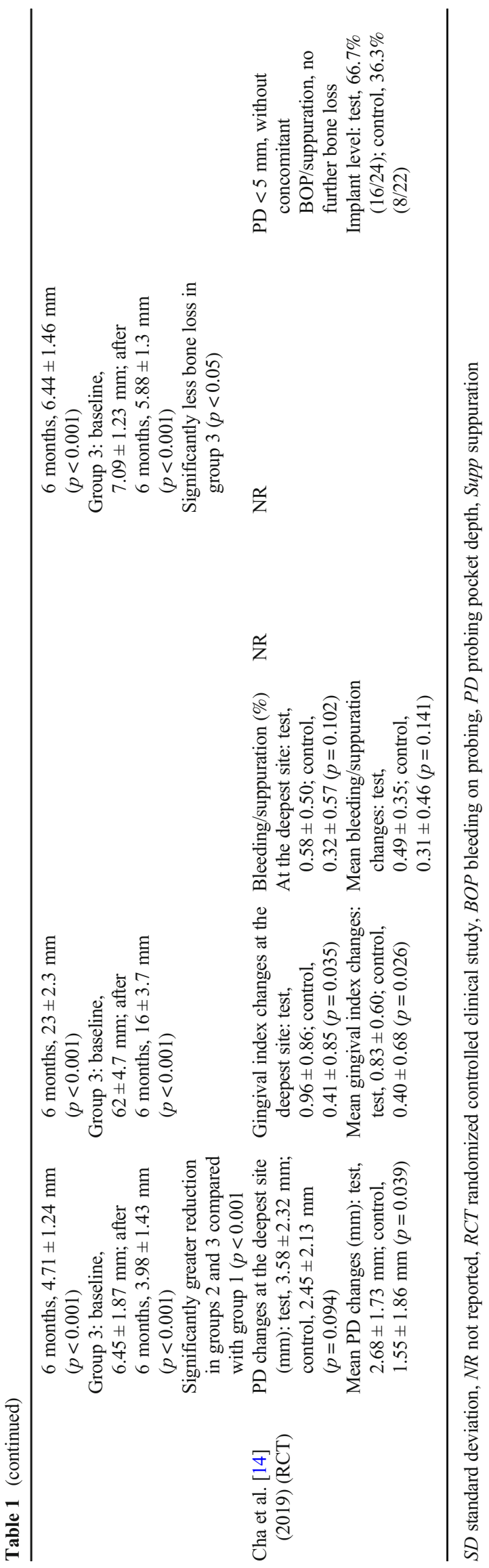

supracrestally exposed rough implant surfaces, which, in turn, would be less prone to plaque accumulation and, ultimately, the recurrence of infection [24, 25, 27-29]. Clinically, the advantage of implantoplasty compared to resective therapy alone in terms of BOP, PD reduction, and marginal bone preservation was demonstrated in a 3-year comparative clinical investigation (Table 2) [24]. Nevertheless, compared to the control sites, adjunctively performed implantoplasty leads to significant postoperative soft tissue recession (1.64 $\mathrm{mm}$ vs. $1.94 \mathrm{~mm}$, respectively) [24].

\section{Outcomes of the Therapy}

Surgical resective peri-implantitis treatment was found to be effective in reducing signs of peri-implant soft tissue inflammation and decreased probing depths in the short term [16, 30]. According to the similar definitions used by different authors, resective therapy yielded success in $14 \%$ of implants after 6 months [21] and in 75\% of implants after 3 years [26] (Table 2). Over a 5-year period, healthy conditions $(\mathrm{PD}<$ $4 \mathrm{~mm}$ and no BOP or Supp) were found in $60 \%$ of implants for patients enrolled in a 6-month recall system [15].

The characteristics of implant surfaces were demonstrated to be among the prognostic factors for the successful treatment of peri-implantitis following resective treatment [19, 20]. Over 3 years, treatment success (no bone loss $>0.5 \mathrm{~mm}$, PD $\leq 5 \mathrm{~mm}$, and no BOP or Supp) was more frequently observed for implants with nonmodified surfaces compared to implants with modified surfaces (61\% vs. $23 \%$, respectively) [19]. In addition, retrospective data demonstrated significantly higher PD and BOP reduction as well as greater crestal bone preservation for the implants with nonmodified surfaces 2 to 10 years after the resective therapy [31]. Factors, such as the preoperative presence of Supp, bone loss $>7 \mathrm{~mm}$, and PD $>8 \mathrm{~mm}$, as well as residual PDs $\geq 4 \mathrm{~mm}$ following resective periimplantitis therapy, were found to be associated with the reduced therapeutic success and increased the risk of further disease progression $[15,21]$.

\section{Augmentative Approaches}

\section{Augmentative Therapy}

In addition to resolving infection, augmentative therapy for peri-implantitis is aimed at (1) regenerating the bone defect, (2) achieving re-osseointegration, and (3) limiting the recession of peri-implant soft tissue [32]. Indications of this surgical approach involve the presence of intrabony defects with a minimum depth of $3 \mathrm{~mm}$, three- or four-wall-contained defects, and the presence of keratinized mucosa [32]. 
Fig. 1 A clinical case of resective peri-implantitis therapy combined with implantoplasty. a Presurgical view following the removal of a suprastructure. b Radiographic view. Marginal bone loss present around implants 14 and 16. c Implantoplasty performed at supracrestally exposed implant sites. d Suturing. e Intraoral view after 6 months
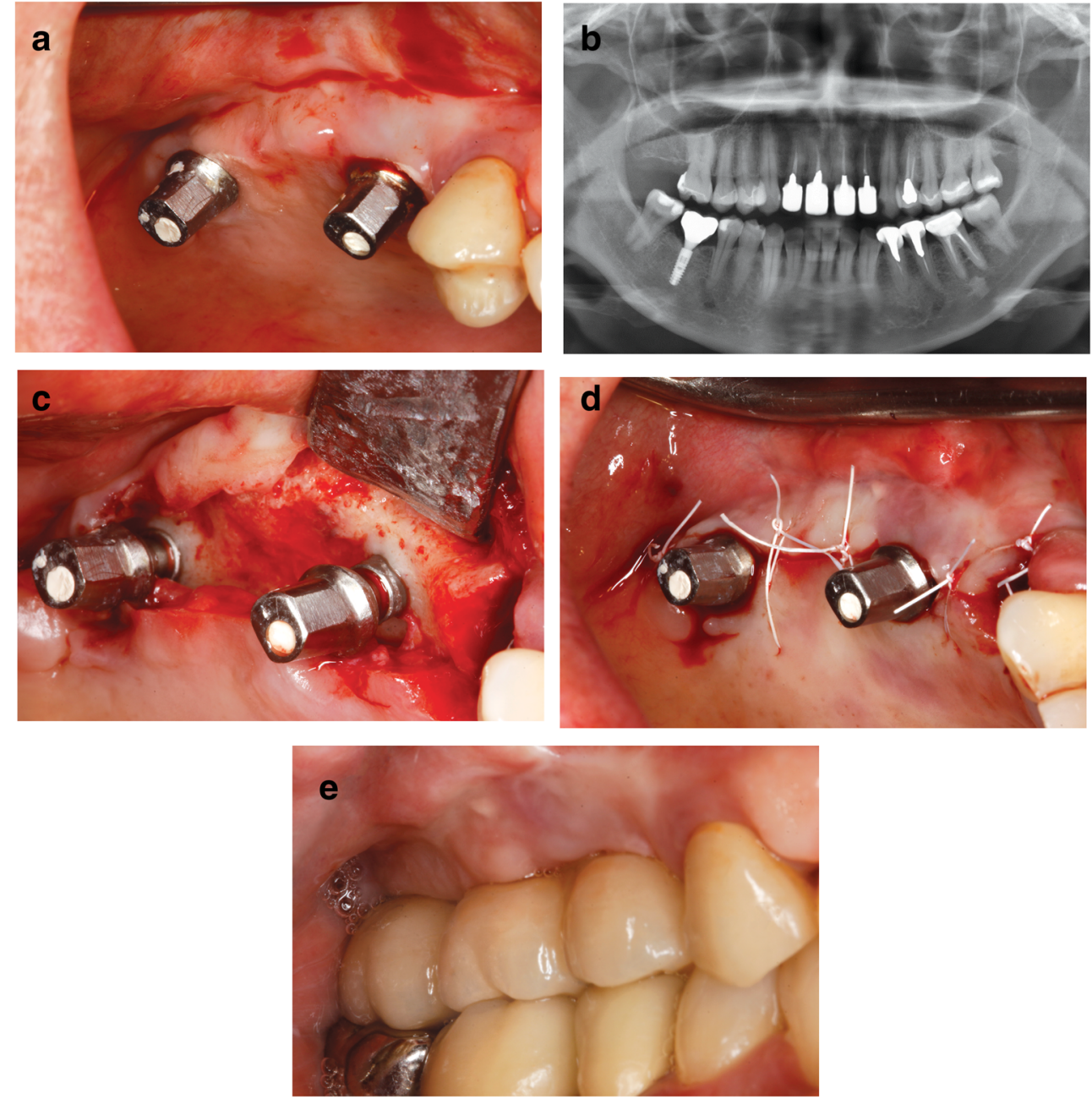

\section{Interventions}

Augmentative peri-implantitis treatment protocol includes the following steps (Fig. 2):

- Access to the defect

- Removal of inflamed tissues

- Decontamination of the implant surface

- Placement of the graft material (with or without a barrier membrane)

- Adequate flap adaptation

Implant surface decontamination methods applied during augmentative peri-implantitis treatment include mechanical debridement (i.e., plastic, titanium, or carbon curettes; titanium brushes; or air polishing), laser therapy (carbon dioxide and Er:YAG lasers), application of chemical agents (chlorhexidine digluconate, citric acid, minocycline, sterile saline, ethylenediaminetetraacetic acid (EDTA) 24\%, or hydrogen peroxide), and their combinations (Table 3 ) $[8,33-38,40]$. As indicated in a recent systematic review, currently existing clinical, radiographic, and microbiological data do not favor any implant surface decontamination approach and fail to show the influence of a particular decontamination protocol on the long-term outcomes of peri-implantitis surgical therapy [41].

The impact of such factors as the healing pattern (i.e., submerged or nonsubmerged) or additional use of systemic antibiotics for the therapeutic outcomes of augmentative periimplantitis therapy cannot be investigated because of the absence of comparative studies [42]. Nevertheless, whenever feasible, to facilitate protected physiological healing, clinicians are recommended to choose submerged postoperative wound closure [43].

\section{Grafting Materials}

Reconstruction of peri-implant bone defect can be performed by either the use of bone substitute material solely or in combination with a barrier membrane [42]. Bone replacement materials suggested for peri-implant bone defect fill include 


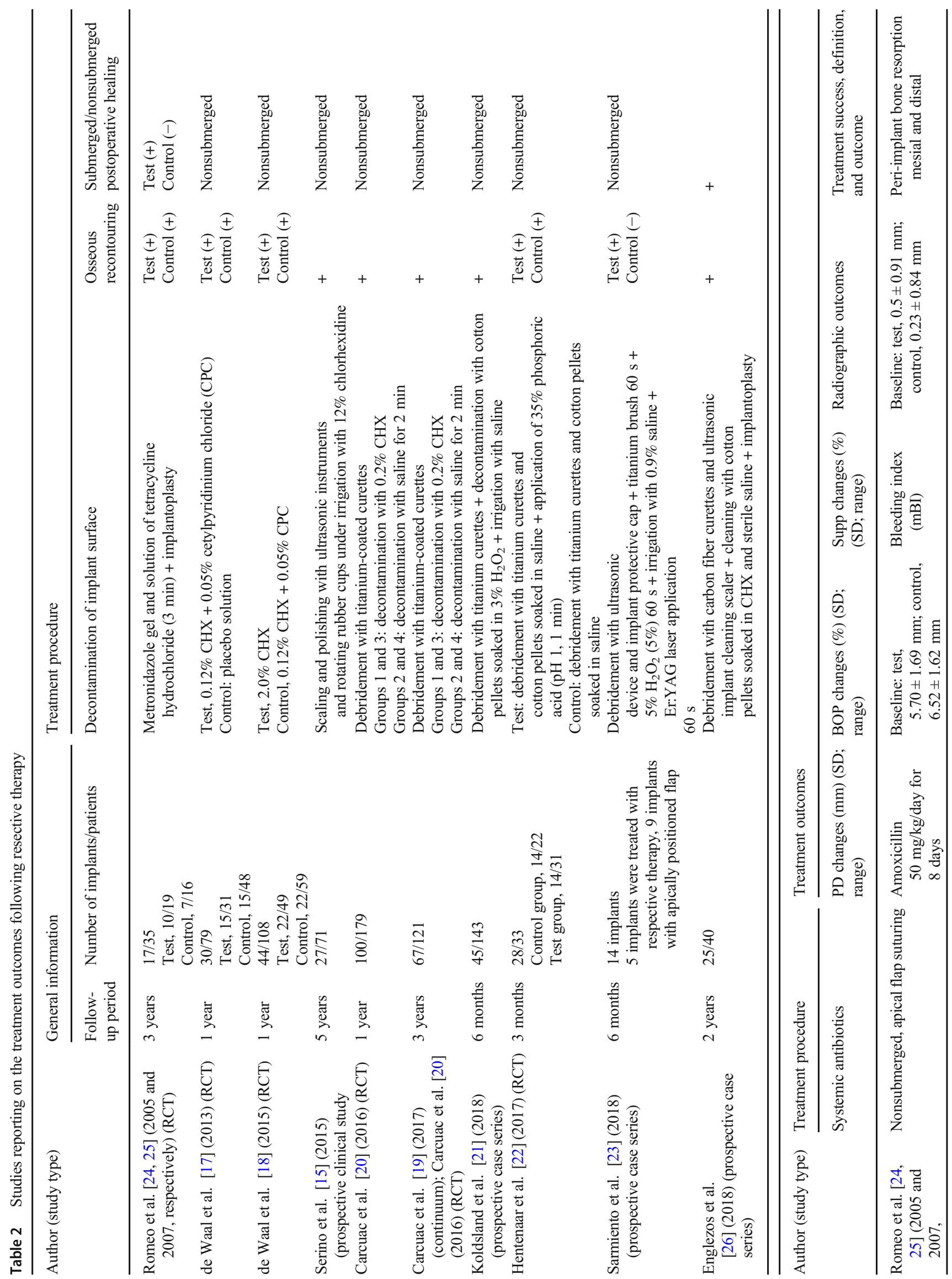




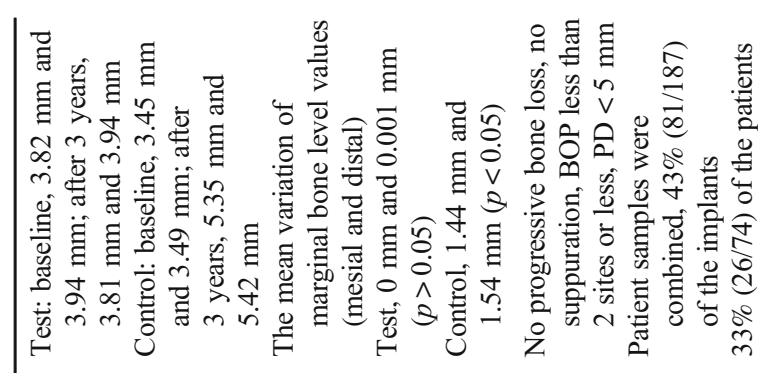

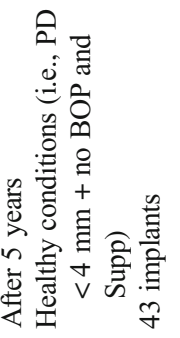

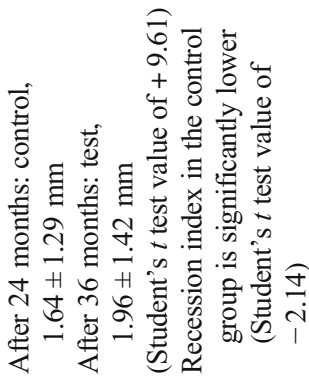

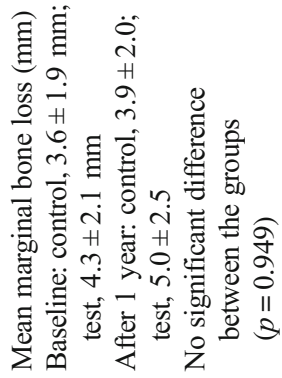

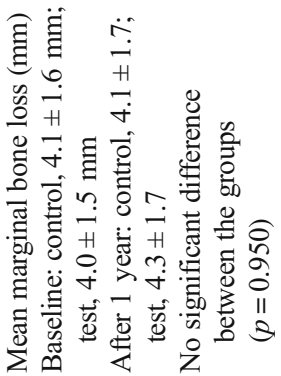

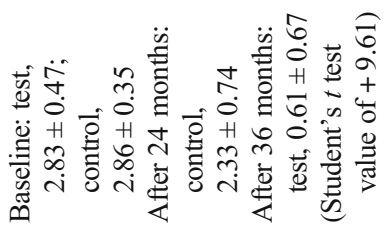

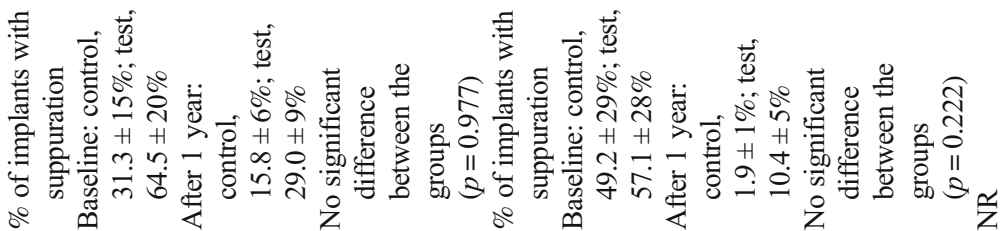

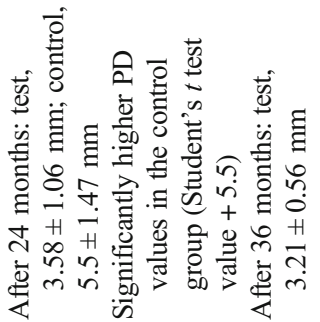

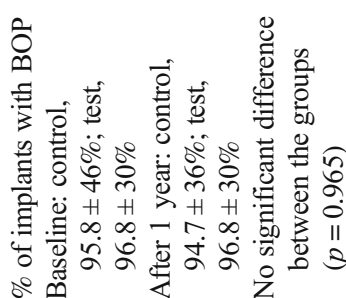

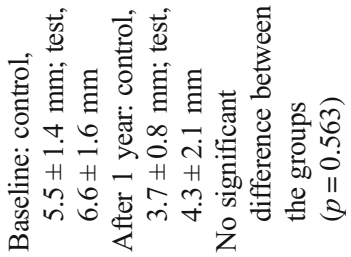

萬

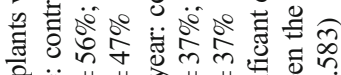

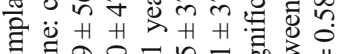

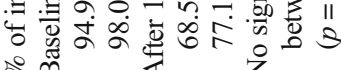

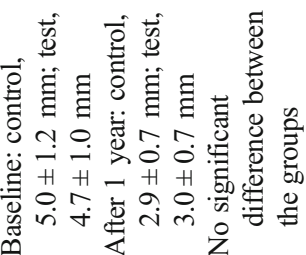

亮
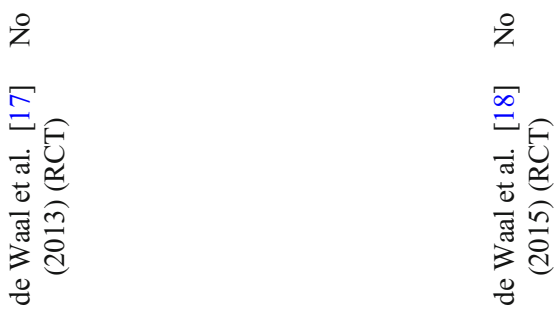

z̊

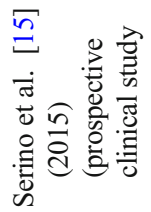




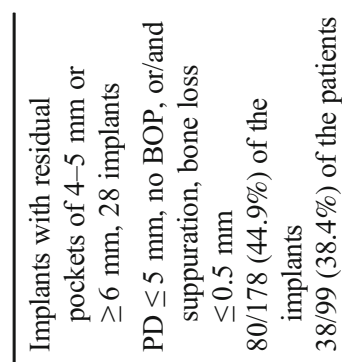

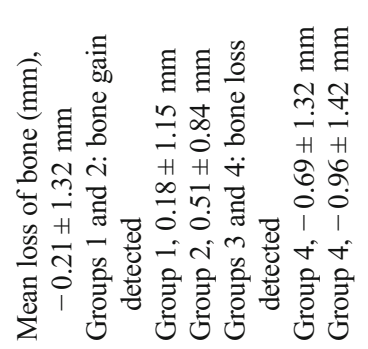

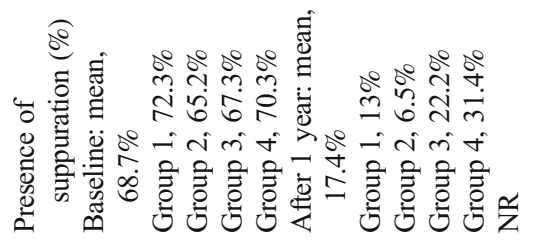

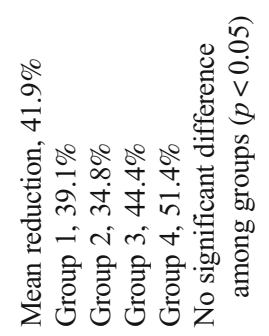

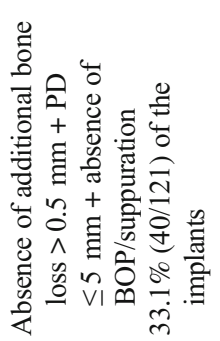

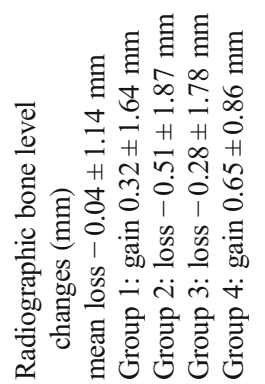

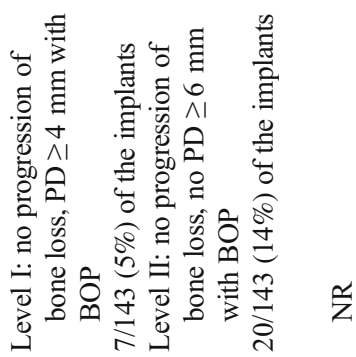

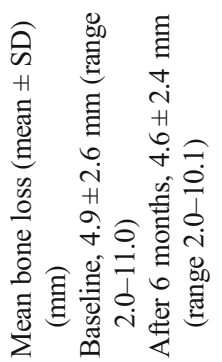

兴
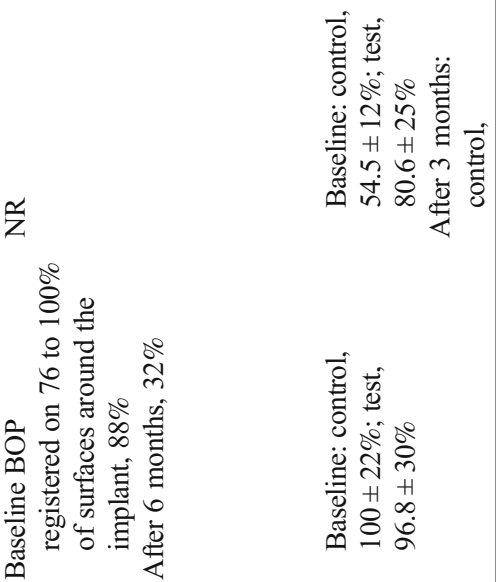

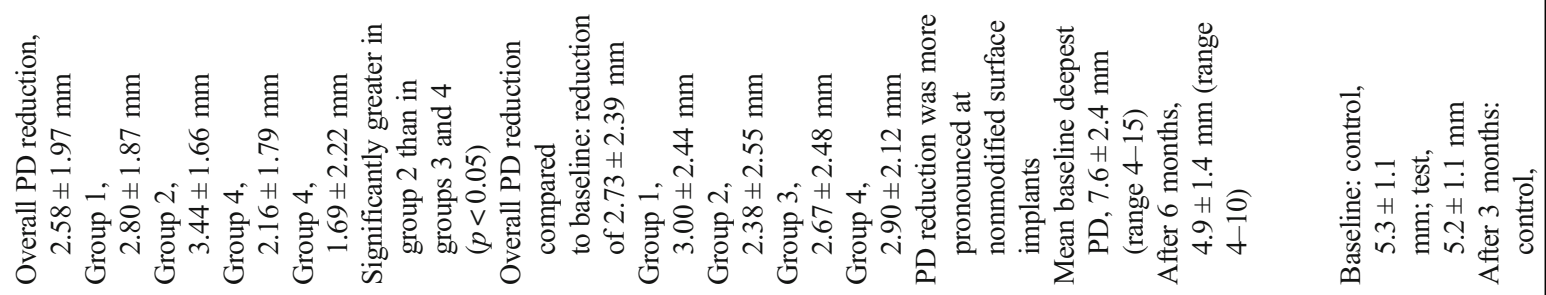

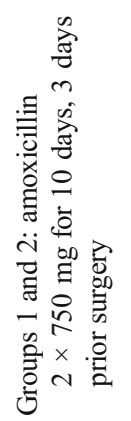

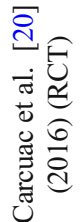

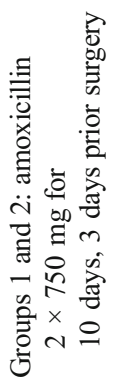

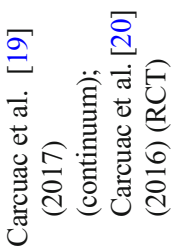

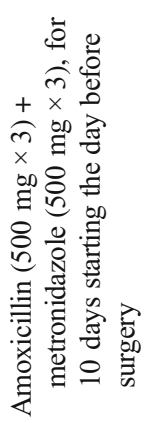

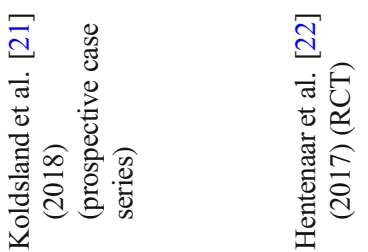


292

Gur Oral Health Rep (2020) 7:283-303

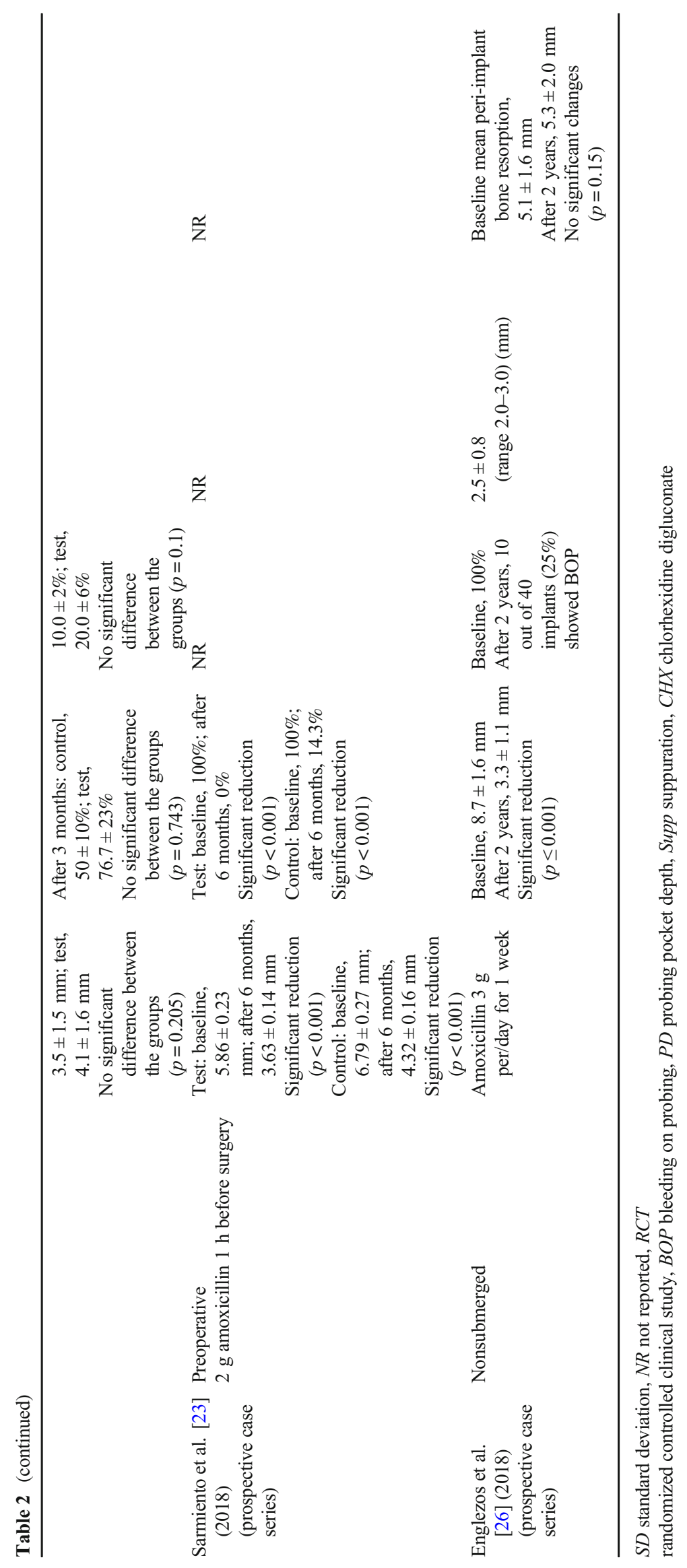

Springer 
Fig. 2 Peri-implantitis therapy with an augmentative approach. a Intrabony circumferential defect at implant 46. b Intrabony defect fill with bone substitute and coverage with collagen membrane. c Suturing. d Intraoral view after 12 months
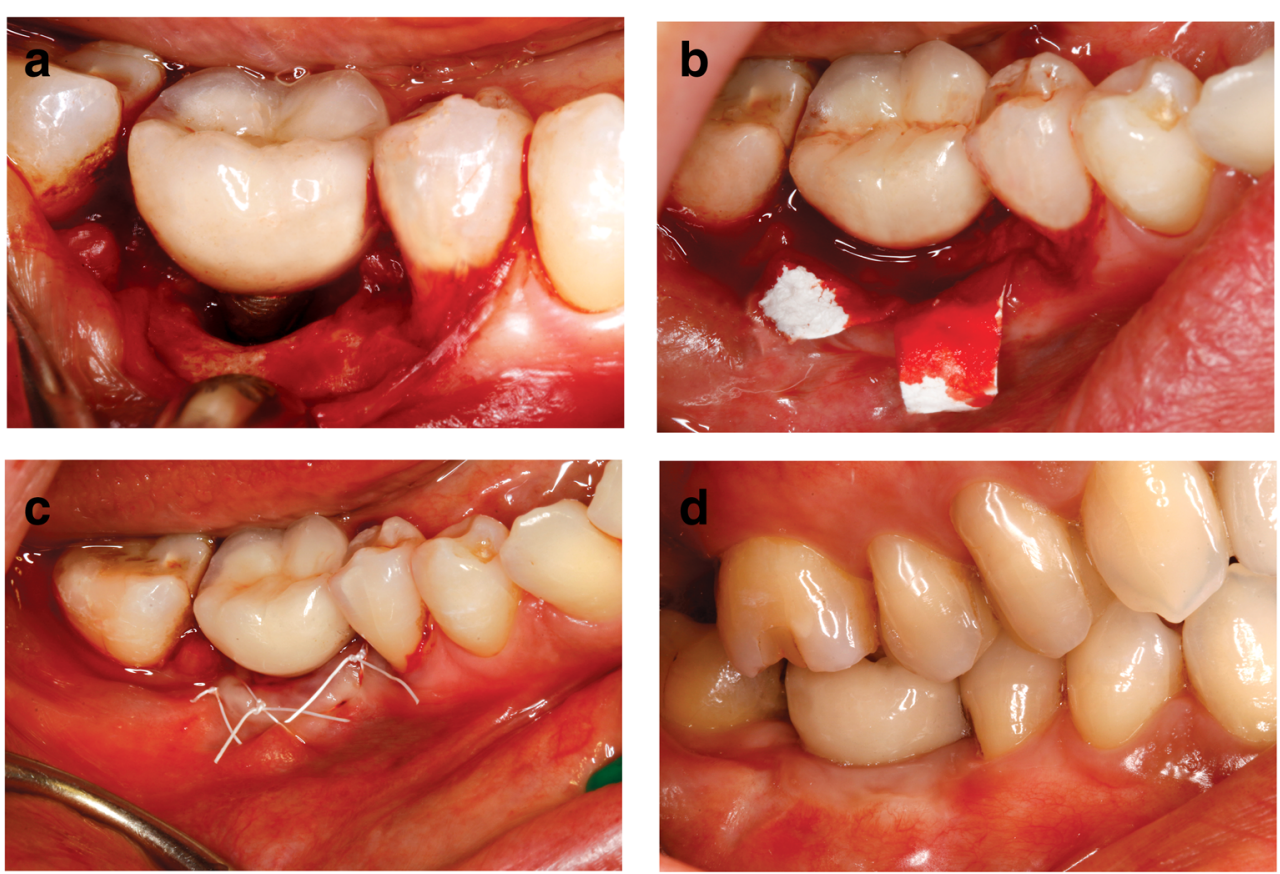

autogenous bone; alloplastic, xenogenic, and allogenic bone substitutes; and titanium granules [8, 33-35, 37, 38, 40, 44].

Findings of a comparative investigation pointed toward significantly better clinical and radiographic outcomes when using a xenograft over autogenous bone [34]. Superior clinical treatment outcomes were obtained at the implant sites where xenogeneic bone substitute was applied over alloplastic bone particles (i.e., hydroxyapatite) [33]. Furthermore, a significantly higher radiographic fill of peri-implant bone defect resulted was noted at the implant sites treated with titanium particles compared to the xenogenic bone substitute, although the clinical outcomes (i.e., PD and BOP changes) did not differ between the two treatment modalities [40]. It should be, however, elucidated that the findings of the aforementioned comparative investigations should be evaluated with caution since the compared bone fill materials (i.e., autogenous bone vs. xenograft, xenograft vs. titanium granules) exhibit different opacity properties, which may lead to the misinterpretation of the data.

The potential beneficial effect of the application of enamel matrix derivates (EMDs) into intrabony peri-implant defects of $\geq 3 \mathrm{~mm}$ depth was investigated over a 5-year period [45, 46]. After 1 year, significantly higher marginal bone levels and an increased prevalence of Gram-positive and Gramnegative aerobic bacteria compared with OFD alone were noted at the implant test sites [45]. However, over the 3-year and 5 -year periods, the positive effect associated with the superior marginal bone level was not sustained, as a bone level gain of $1.3 \mathrm{~mm}$ was observed in the control group, and a gain of $1.4 \mathrm{~mm}$ was noted in the test group, with no significant difference between the two groups $(p=0.043)$ [46].
Conflicting data exist with regard to the rationale for using barrier membranes to improve augmentative treatment outcomes [33, 35, 44]. In particular, two studies did not observe beneficial effects from the adjunctive use of a barrier membrane over autogenous bone or alloplastic bone substitute alone after 3 years and 5 years, respectively [35, 44]. Contrarily, over a 4-year period, the use of a combination of xenogenic bone and a collagen membrane provided better clinical outcomes, in terms of BOP and $\mathrm{PD}$ reduction, than hydroxyapatite particles alone [33].

\section{Outcomes of the Therapy}

The overall efficacy of augmentative peri-implantitis therapy was assessed in a recent systematic review and meta-analysis [47]. Based on its findings, significant improvement in marginal bone levels (weighted mean difference $(\mathrm{WMD})=2.0 \mathrm{~mm}$ ), clinical attachment gain $(\mathrm{WMD}=1.8 \mathrm{~mm})$, and reduction of the $\mathrm{PD}$ values $(\mathrm{WMD}=2.8 \mathrm{~mm})$ were obtained at peri-implantitis sites treated with adjunctive-augmentative measures [47]. Assessment of the potential beneficial effects of the augmentative therapy over the controls (i.e., open flap debridement) showed a significantly higher gain in marginal bone levels of $1.7 \mathrm{~mm}$ and defect fill $(\mathrm{WMD}=57 \%$ ), favoring augmentative measures [47]. Clinically, augmentative therapy resulted in significant postoperative soft tissue recession (WMD $=0.7 \mathrm{~mm}$ ), and when compared to the control sites, the therapy failed to produce significant reductions in PD and BOP values [47].

Peri-implant defect morphology has shown to impact upon the augmentative outcomes for the management of peri-implantitis [48]. Specifically, augmentation of 


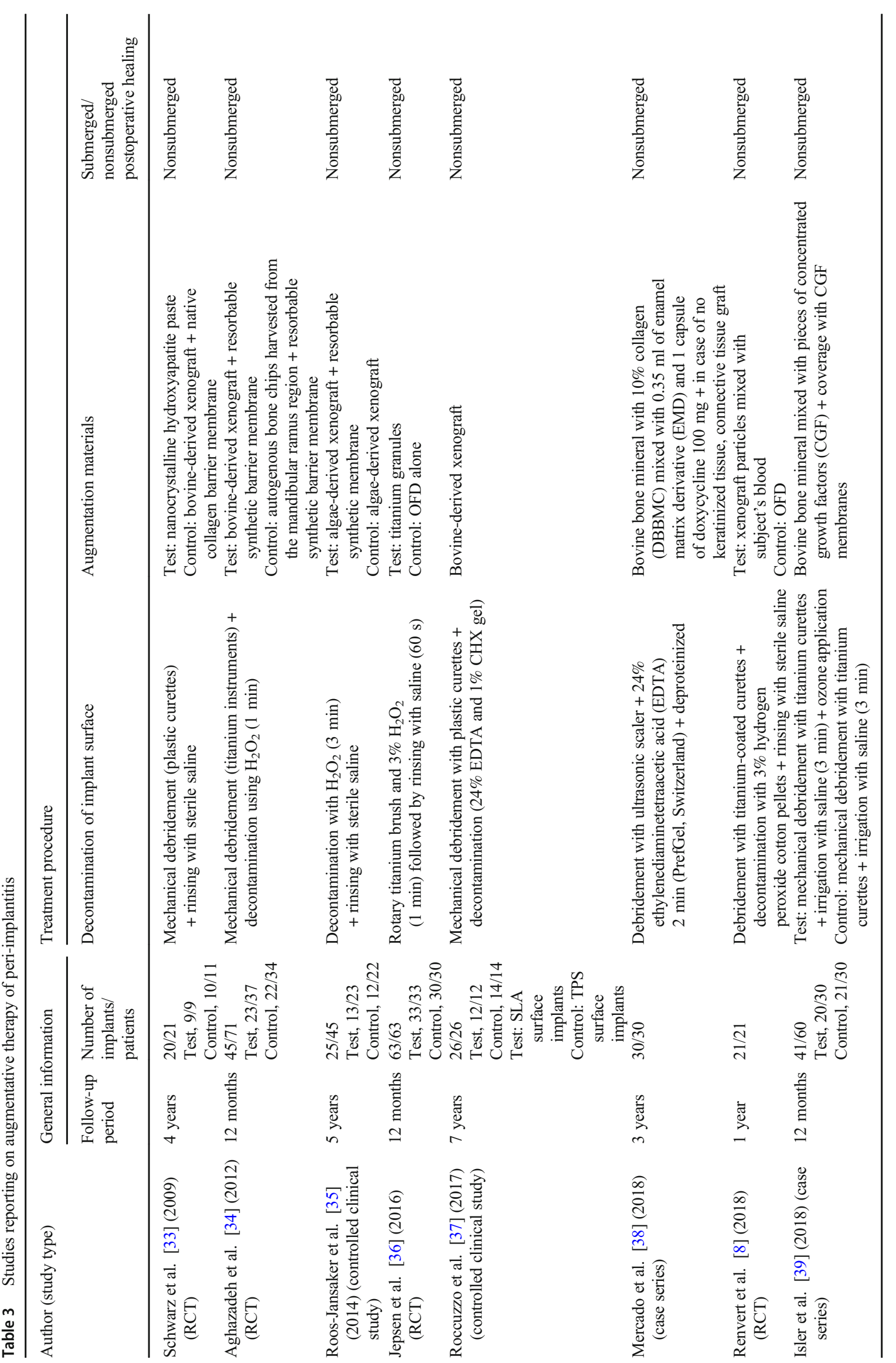




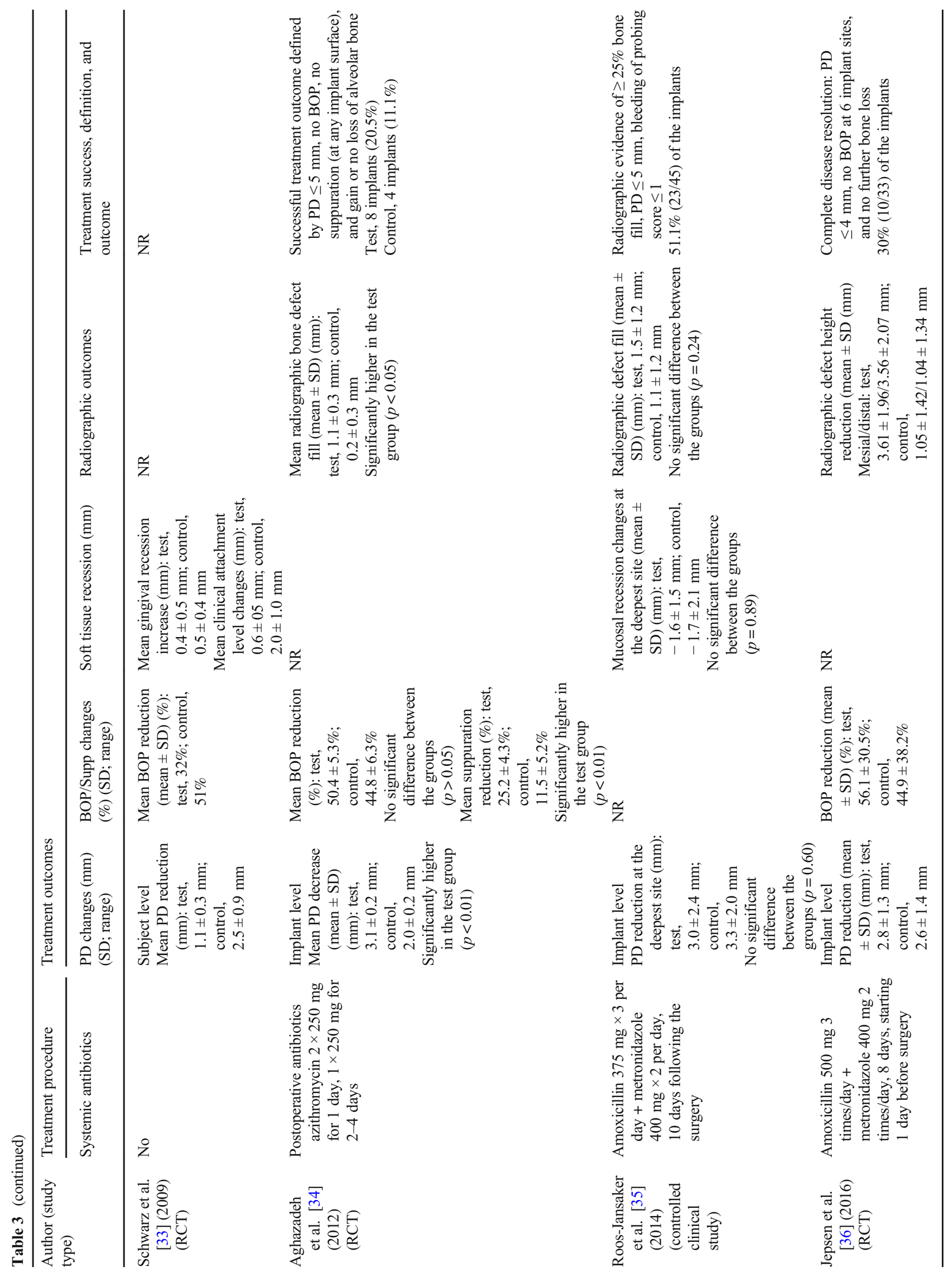




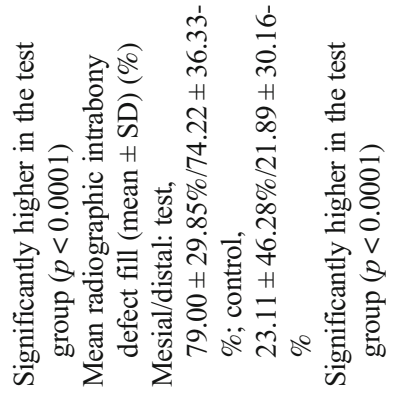

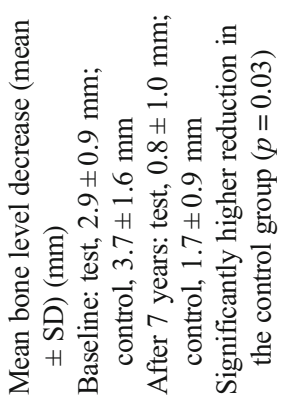

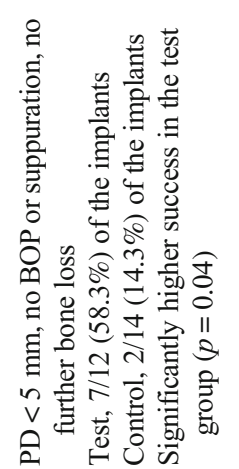
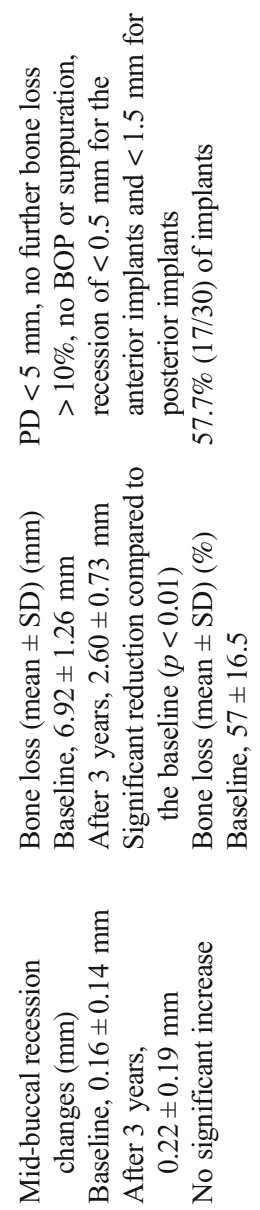

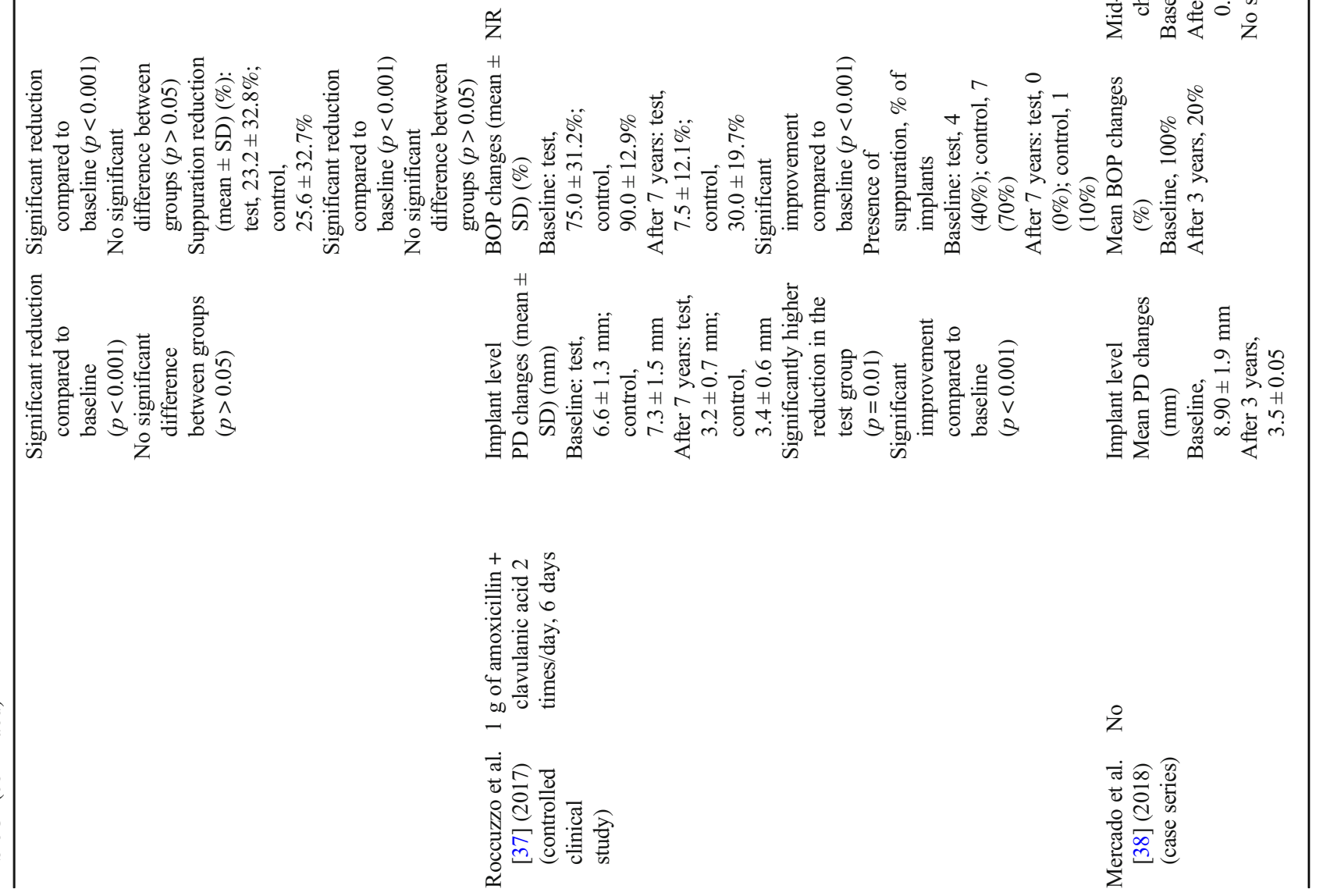



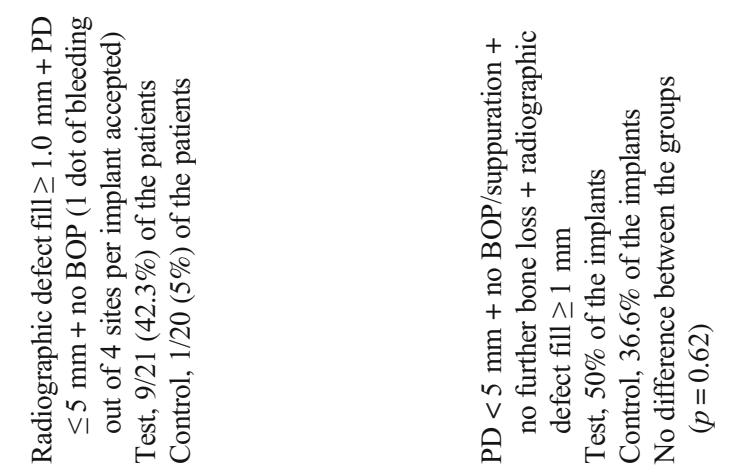

总

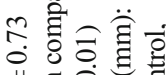

H

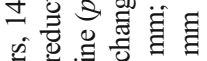

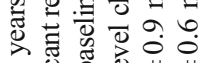

$m$ 造 +1

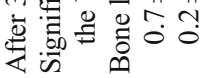

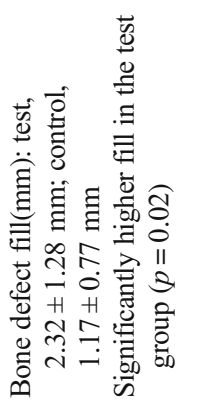

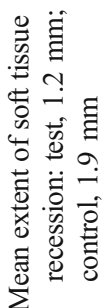

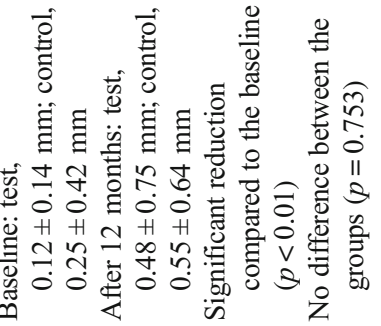

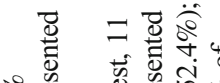

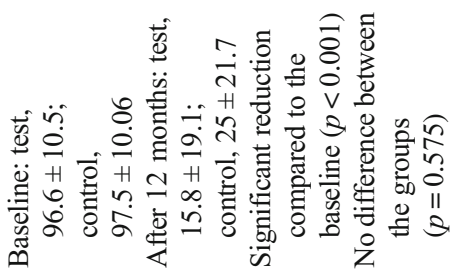

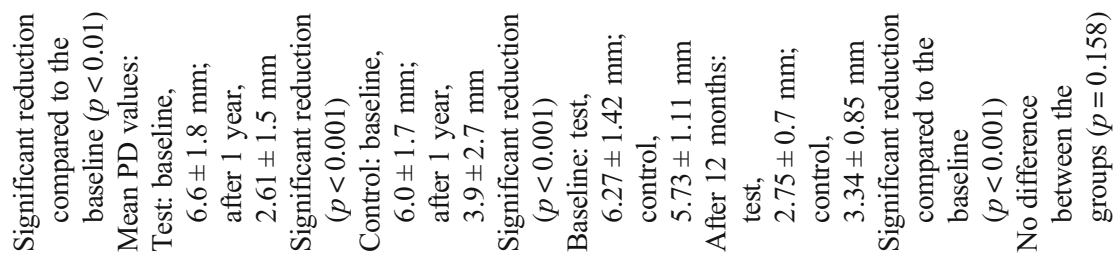

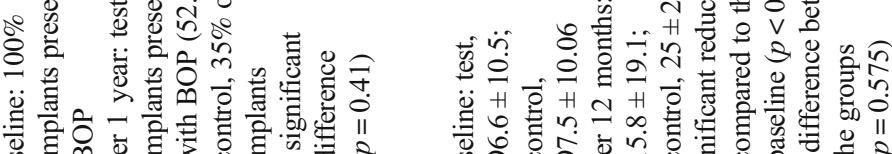

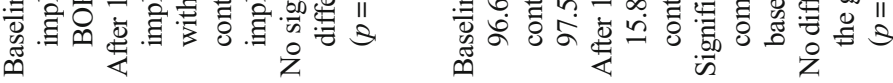
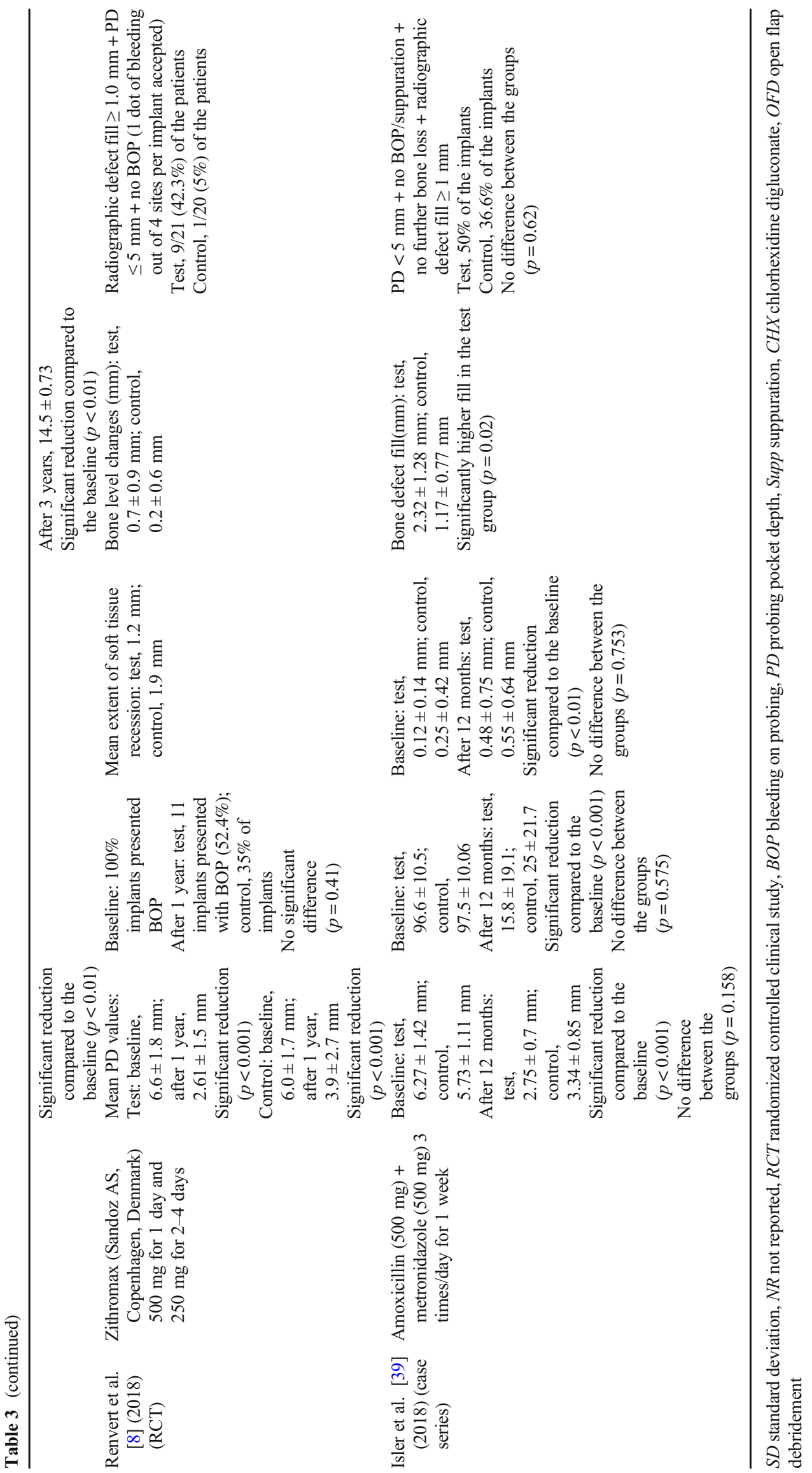

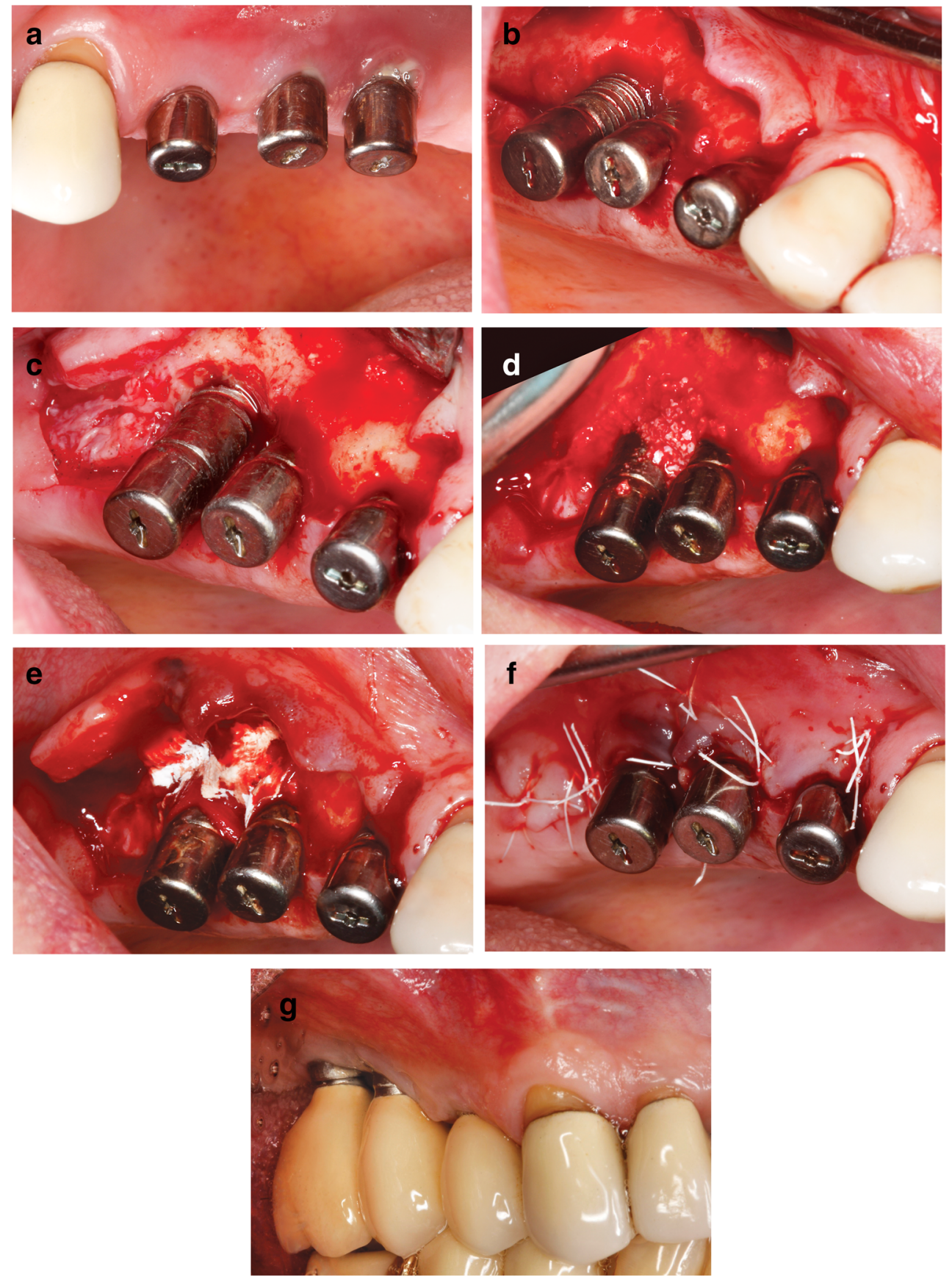

Fig. 3 Combined peri-implantitis therapy. a Presurgical view following the removal of a suprastructure. Suppuration detected at implants placed in regions 15 and 16. b Raising of full-thickness flap and removal of granulation tissues. c Implantoplasty performed at the supracrestally

circumferential peri-implant defects resulted in higher PD reduction and clinical attachment level (CAL) gain at 6 months and 12 months compared with the dehiscencetype defects [48]. Furthermore, treatment success (defined as PD $<5 \mathrm{~mm}$, no BOP or Supp, and no further bone loss) was more frequently achieved for rough-surfaced implants versus moderately rough implants (14\% vs. $58 \%$, respectively) [37]. and buccally exposed implant surfaces. d Intrabony defect at implant 16 filled with bone substitute. e Placement of collagen membrane over the bone substitute. f Suturing. g Clinical view after 6 months

\section{Combined Therapy}

Combined peri-implantitis therapy includes implantoplasty (i.e., mechanical modification of the implant surface) performed at implant sites where no bone regeneration is expected (i.e., supracrestally and buccally exposed implant parts) followed by augmentation of the intrabony defect components [48]. This surgical approach might be applicable in the majority of peri- 


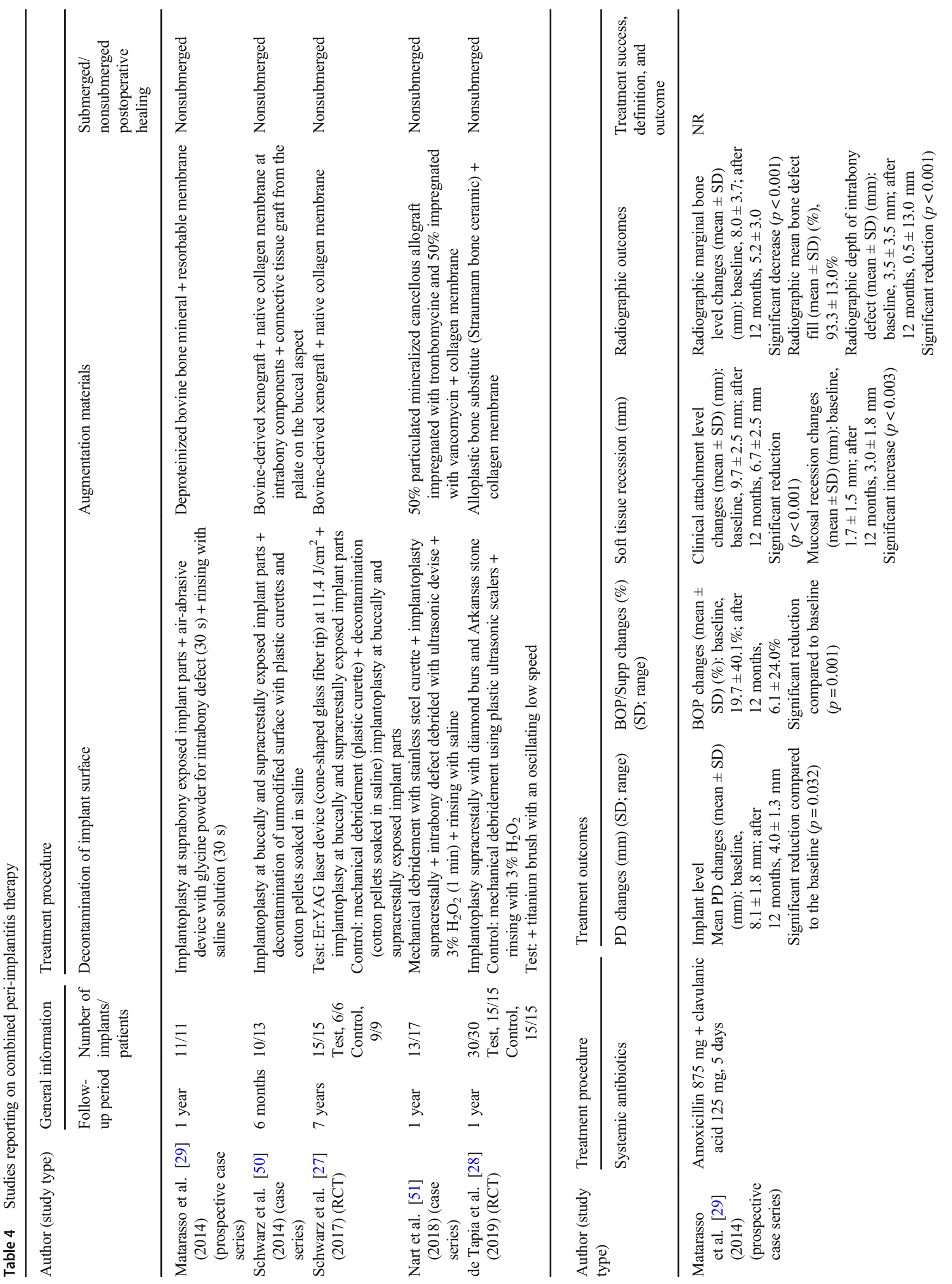


艺

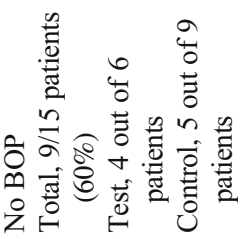

岂

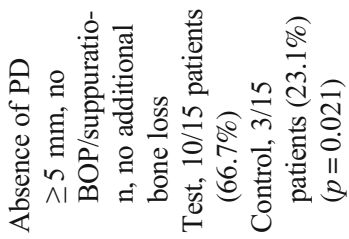

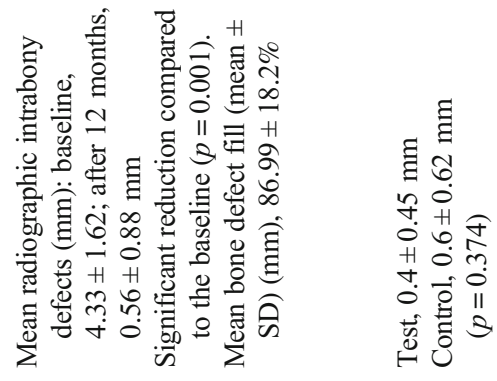

号

兑

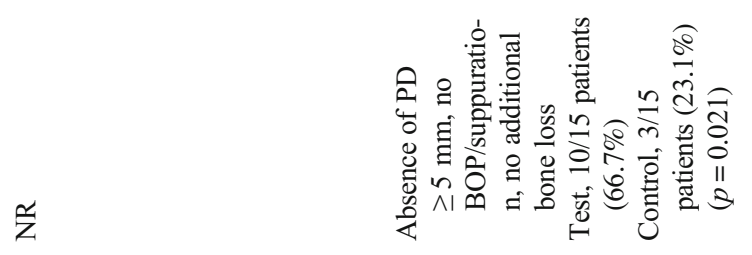

त)

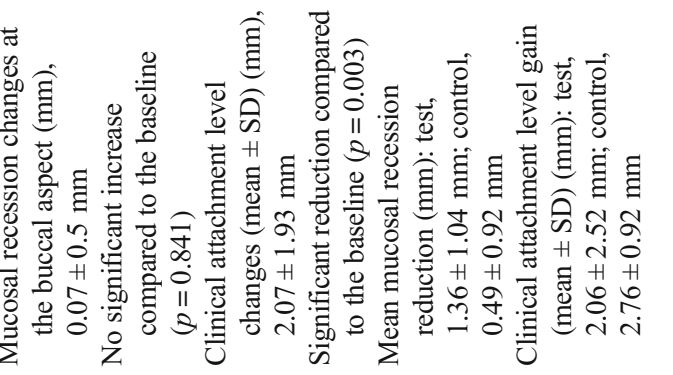

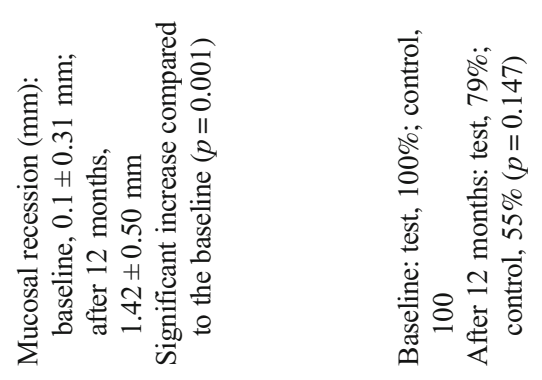

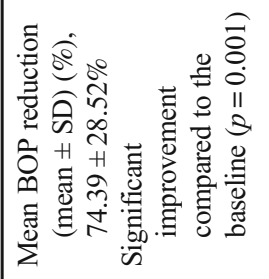

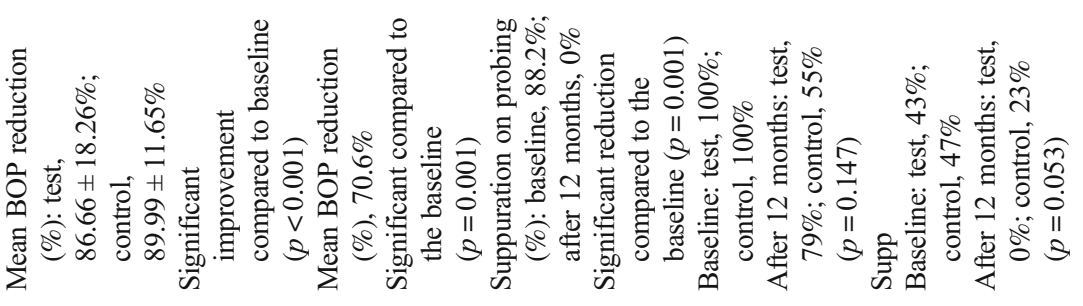

볼

宩卉 离

此

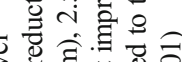

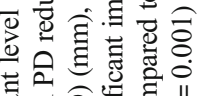

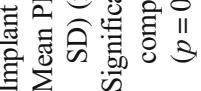
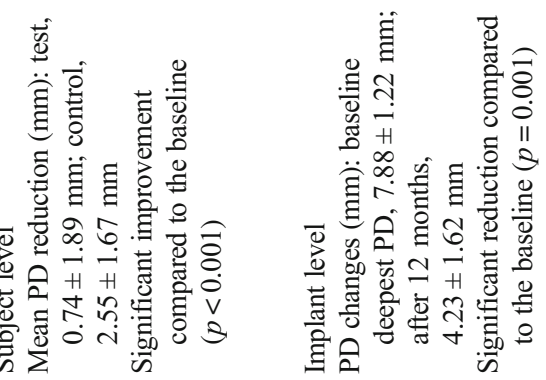

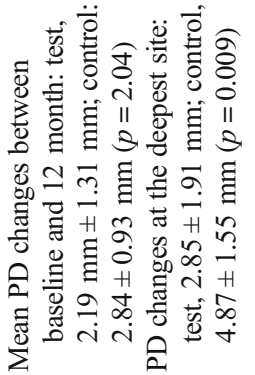

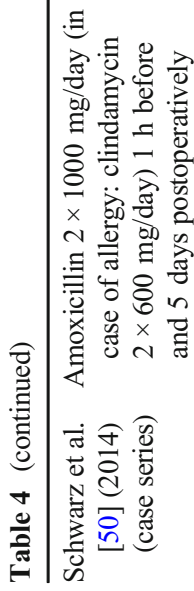
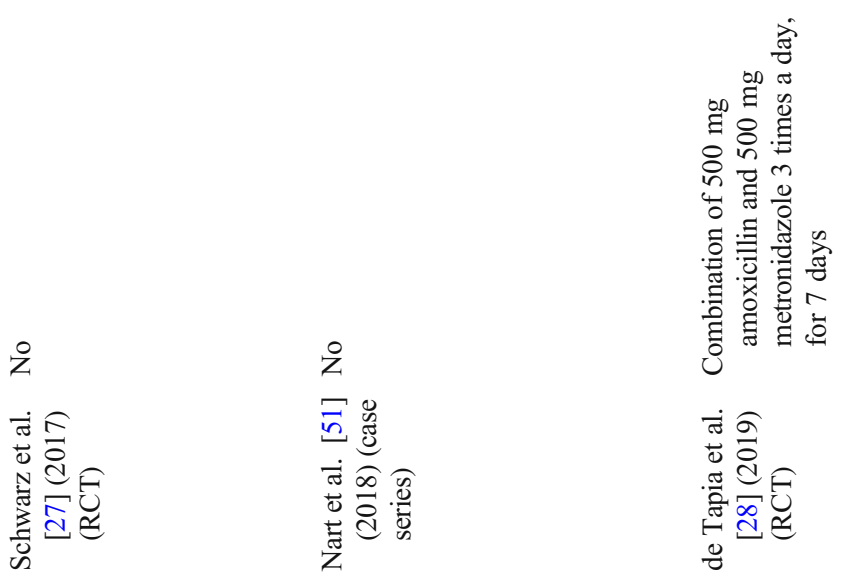

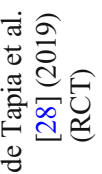


implantitis cases, as more than half of naturally occurring periimplantitis sites $(79 \%)$ have a combined configuration, including intrabony and suprabony components [49].

\section{Procedure}

Combined peri-implantitis surgery comprises the following steps (Fig. 3):

- Access to the defect

- Removal of inflamed tissues

- Decontamination of the implant surface

- Implantoplasty performed at buccally and supracrestally exposed implant parts

- Grafting of the intrabony defect (bone substitute + barrier membrane) with or without a connective tissue graft

- Adequate flap adaptation

With respect to the method of implant surface decontamination, over a 12-month period, the use of titanium brushes (i.e., mechanical debridement with an ultrasonic scaler + rinsing with $3 \% \mathrm{H}_{2} \mathrm{O}_{2}+$ titanium brush) led to significantly better PD reduction $(4.87 \mathrm{~mm}$ vs. $2.85 \mathrm{~mm})$ and bone defect fill (2.61 $\mathrm{mm}$ vs. $1.17 \mathrm{~mm}$ ) compared with the controls (i.e., mechanical debridement with ultrasonic scaler + rinsing with $3 \%$ $\mathrm{H}_{2} \mathrm{O}_{2}$ ) [28] (Table 4). Contrarily, during a 7-year follow-up period, Er:YAG laser application showed similar treatment outcomes to debridement with plastic curettes and cotton pellets soaked in sterile saline [27].

For the reconstruction of the intrabony defects, xenogenic bone substitute or antibiotic impregnated allograft was used in conjunction with collagen membrane (Table 4) [29, 50, 51]. A concomitant soft tissue augmentation with a concomitant connective tissue graft placed on the buccal aspect performed with a combined peri-implantitis therapy after 6 months led to a mean gain in facial soft tissue height of $0.07 \mathrm{~mm}$ around 13 implant sites [50].

\section{Outcomes of the Therapy}

Compared with the baseline, combined peri-implantitis therapy significantly reduces BOP, PD, and Supp [27, 29, 51]. A significant reduction in intrabony defects, with a mean radiographic intrabony defect fill of 87 to $93 \%$, was detected after 1 to 7 years $[29,51]$. The rate of successful treatment (defined as the absence of $\mathrm{PD} \geq 5 \mathrm{~mm}$, no BOP/Supp, and no additional bone loss) was significantly higher for the patients whom titanium brushes were adjunctively used for decontaminating the implant surface $(66.7 \%(10 / 15)$ vs. $23.1 \%(3 / 15))$ [28]. Peri-implant tissue health (i.e., absence of BOP) was detected in $60 \%(9 / 15)$ of the patients 7 years after the combined therapy [27]. As indicated by the retrospective data, disease resolution (i.e., the absence of $\mathrm{BOP}$ and $\mathrm{PD} \geq 6 \mathrm{~mm}$ ) was obtained in $28 \%(11 / 39)$ of the patients at 6 months to 10 years following combined peri-implantitis therapy [52].

\section{Summary}

The disease severity, the defect's regenerative potential, and patient expectations should be evaluated prior to choosing a surgical technique for peri-implantitis management. OFD with or without adjunctive resective measures may be indicated in the presence of horizontal bone loss. Implantoplasty as a part of periimplantitis surgical therapy may improve soft tissue inflammatory status; however, it can lead to more extensive mucosal recession. At peri-implantitis sites exhibiting intrabony defects, augmentative measures should be favored. In more advanced cases with combined defect configurations, a combination of augmentative and resective measures may be feasible. Soft tissue volume grafting as an adjunct to surgical peri-implant therapy may be effective to overcome mucosa recession in the aesthetic zone.

Funding Information Open Access funding provided by Projekt DEAL.

\section{Compliance with Ethical Standards}

Conflict of Interest The authors declare that they have no conflict of interest.

Human and Animal Rights and Informed Consent This article does not contain any studies with human or animal subjects performed by any of the authors.

Open Access This article is licensed under a Creative Commons Attribution 4.0 International License, which permits use, sharing, adaptation, distribution and reproduction in any medium or format, as long as you give appropriate credit to the original author(s) and the source, provide a link to the Creative Commons licence, and indicate if changes were made. The images or other third party material in this article are included in the article's Creative Commons licence, unless indicated otherwise in a credit line to the material. If material is not included in the article's Creative Commons licence and your intended use is not permitted by statutory regulation or exceeds the permitted use, you will need to obtain permission directly from the copyright holder. To view a copy of this licence, visit http://creativecommons.org/licenses/by/4.0/.

\section{References}

Papers of particular interest, published recently, have been highlighted as:

- Of importance

•. Of major importance

1. Berglundh T, et al. Peri-implant diseases and conditions: consensus report of workgroup 4 of the 2017 world workshop on the classification of periodontal and Peri-implant diseases and conditions. $\mathrm{J}$ 
Periodontol. 2018;89(Suppl 1):S313-s318 This manuscript is a part of the World Workshop on the Classification of Periodontal and Peril-Implant Diseases and Conditions (2017), and presents classifications and diagnostic criteria for peri-implant diseases.

2.• Schwarz F, et al. Peri-implantitis. J Periodontol. 2018;89(Suppl 1): S267-s290 This manuscript provides a current update on etiology, clinical characteristics, and risk factors/indicators for periimplantitis.

3.• Derks J, et al. Peri-implantitis - onset and pattern of progression. J Clin Periodontol. 2016;43(4):383-8 This clinical analysis evaluates the clinical characteristics of peri-implant diseases that are of great relevance in a clinical practice.

4. Derks J, et al. Effectiveness of implant therapy analyzed in a Swedish population: prevalence of Peri-implantitis. J Dent Res. 2016;95(1):43-9.

5. Sanz M, Chapple IL. Clinical research on peri-implant diseases: consensus report of working group 4. J Clin Periodontol. 2012;39(Suppl 12):202-6.

6. Ramanauskaite A, Daugela P, Juodzbalys G. Treatment of periimplantitis: meta-analysis of findings in a systematic literature review and novel protocol proposal. Quintessence Int. 2016;47(5): 379-93.

7. Klinge B, et al. Peri-implant diseases. Eur J Oral Sci. 2018;126(Suppl 1):88-94.

8. Renvert S, Roos-Jansaker AM, Persson GR. Surgical treatment of peri-implantitis lesions with or without the use of a bone substitutea randomized clinical trial. J Clin Periodontol. 2018;45(10):126674.

9. Lang NP, Salvi GE, Sculean A. Nonsurgical therapy for teeth and implants-when and why? Periodontol. 2019;79(1):15-21.

10.• Heitz-Mayfield LJA, et al. Supportive peri-implant therapy following anti-infective surgical peri-implantitis treatment: 5 -year survival and success. Clin Oral Implants Res. 2018;29(1):1-6 This is a clinical study evaluates the mid-term (5-years) peri-implantitis surgical treatment success among the patients enrolled in a regular supportive therapy.

11. Serino G, Turri A, Lang NP. Maintenance therapy in patients following the surgical treatment of peri-implantitis: a 5-year follow-up study. Clin Oral Implants Res. 2015;26(8):950-6.

12. Hallstrom $\mathrm{H}$, et al. Open flap debridement of peri-implantitis with or without adjunctive systemic antibiotics: a randomized clinical trial. J Clin Periodontol. 2017;44(12):1285-93.

13. Toma S, Brecx MC, Lasserre JF. Clinical evaluation of three surgical modalities in the treatment of Peri-Implantitis: a randomized controlled clinical trial. J Clin Med. 2019;8(7):966.

14. Cha JK, Lee JS, Kim CS. Surgical therapy of Peri-Implantitis with local minocycline: a 6-month randomized controlled clinical trial. J Dent Res. 2019;98(3):288-95.

15. Papadopoulos CA, et al. The utilization of a diode laser in the surgical treatment of peri-implantitis. A randomized clinical trial. Clin Oral Investig. 2015;19(8):1851-60.

16. Keeve PL, et al. Surgical treatment of Periimplantitis with nonaugmentative techniques. Implant Dent. 2019;28(2):177-86.

17. de Waal YC, et al. Implant decontamination during surgical periimplantitis treatment: a randomized, double-blind, placebo-controlled trial. J Clin Periodontol. 2013;40(2):186-95.

18. de Waal YC, et al. Implant decontamination with $2 \%$ chlorhexidine during surgical peri-implantitis treatment: a randomized, doubleblind, controlled trial. Clin Oral Implants Res. 2015;26(9):1015-23.

19. Carcuac O, et al. Surgical treatment of peri-implantitis: 3-year results from a randomized controlled clinical trial. J Clin Periodontol. 2017;44(12):1294-303.

20. Carcuac O, et al. Adjunctive systemic and local antimicrobial therapy in the surgical treatment of Peri-implantitis: a randomized controlled clinical trial. J Dent Res. 2016;95(1):50-7.
21. Koldsland OC, Wohlfahrt JC, Aass AM. Surgical treatment of periimplantitis: prognostic indicators of short-term results. J Clin Periodontol. 2018;45(1):100-13.

22. Hentenaar DFM, et al. Implant decontamination with phosphoric acid during surgical peri-implantitis treatment: a RCT. Int J Implant Dent. 2017;3(1):33.

23. Sarmiento HL, et al. Surgical alternatives for treating Periimplantitis. Int J Periodontics Restorative Dent. 2018;38(5):66571.

24. Romeo E, et al. Therapy of peri-implantitis with resective surgery. A 3-year clinical trial on rough screw-shaped oral implants. Part I: clinical outcome. Clin Oral Implants Res. 2005;16(1):9-18.

25. Romeo E, et al. Therapy of peri-implantitis with resective surgery. A 3-year clinical trial on rough screw-shaped oral implants. Part II: radiographic outcome. Clin Oral Implants Res. 2007;18(2):179-87.

26. Schwarz F, et al. Combined surgical therapy of advanced periimplantitis evaluating two methods of surface decontamination: a 7-year follow-up observation. J Clin Periodontol. 2017;44(3):33742.

27. de Tapia B, et al. The adjunctive effect of a titanium brush in implant surface decontamination at peri-implantitis surgical regenerative interventions: a randomized controlled clinical trial. J Clin Periodontol. 2019;46(5):586-96.

28. Matarasso S, et al. Clinical and radiographic outcomes of a combined resective and regenerative approach in the treatment of periimplantitis: a prospective case series. Clin Oral Implants Res. 2014;25(7):761-7.

29. Ramanauskaite A, et al. Surgical non-regenerative treatments for peri-implantitis: a systematic review. J Oral Maxillofac Res. 2016;7(3):e14.

30. Englezos E, et al. Resective treatment of Peri-implantitis: clinical and radiographic outcomes after 2 years. Int J Periodontics Restorative Dent. 2018;38(5):729-35.

31. Berglundh T, Wennstrom JL, Lindhe J. Long-term outcome of surgical treatment of peri-implantitis. A 2-11-year retrospective study. Clin Oral Implants Res. 2018;29(4):404-10.

32. Jepsen $\mathrm{S}$, et al. Regeneration of alveolar ridge defects. Consensus report of group 4 of the 15th European workshop on periodontology on bone regeneration. J Clin Periodontol. 2019;46(Suppl 21):27786.

33. Aghazadeh A, Rutger Persson G, Renvert S. A single-Centre randomized controlled clinical trial on the adjunct treatment of intrabony defects with autogenous bone or a xenograft: results after 12 months. J Clin Periodontol. 2012;39(7):666-73.

34. Roos-Jansaker AM, et al. Surgical treatment of peri-implantitis using a bone substitute with or without a resorbable membrane: a 5-year follow-up. J Clin Periodontol. 2014;41(11):1108-14.

35. Jepsen K, et al. Reconstruction of Peri-implant osseous defects: a Multicenter randomized trial. J Dent Res. 2016;95(1):58-66.

36. Schwarz F, et al. Surgical regenerative treatment of peri-implantitis lesions using a nanocrystalline hydroxyapatite or a natural bone mineral in combination with a collagen membrane: a four-year clinical follow-up report. J Clin Periodontol. 2009;36(9):807-14.

37. Roccuzzo M, et al. Surgical treatment of peri-implantitis intrabony lesions by means of deproteinized bovine bone mineral with $10 \%$ collagen: 7-year-results. Clin Oral Implants Res. 2017;28(12): 1577-83.

38. Mercado F, Hamlet S, Ivanovski S. Regenerative surgical therapy for peri-implantitis using deproteinized bovine bone mineral with $10 \%$ collagen, enamel matrix derivative and doxycycline-a prospective 3-year cohort study. Clin Oral Implants Res. 2018;29(6): 583-91.

39. Guler B, et al. The comparison of porous titanium granule and xenograft in the surgical treatment of Peri-Implantitis: a prospective clinical study. Clin Implant Dent Relat Res. 2017;19(2):316-27. 
40. Koo KT, et al. Implant surface decontamination by surgical treatment of Periimplantitis: a literature review. Implant Dent. 2019;28(2):173-6.

41. Ramanauskaite A, et al. Surgical treatment of Periimplantitis with augmentative techniques. Implant Dent. 2019;28(2):187-209.

42. Khoury F, et al. Surgical treatment of peri-implantitis - consensus report of working group 4. Int Dent J. 2019;69(Suppl 2):18-22.

43. Khoury F, Buchmann R. Surgical therapy of peri-implant disease: a 3 -year follow-up study of cases treated with 3 different techniques of bone regeneration. J Periodontol. 2001;72(11):1498-508.

44. Isehed $\mathrm{C}$, et al. Effectiveness of enamel matrix derivative on the clinical and microbiological outcomes following surgical regenerative treatment of peri-implantitis. A randomized controlled trial. J Clin Periodontol. 2016;43(10):863-73.

45. Isehed C, et al. Surgical treatment of peri-implantitis using enamel matrix derivative, an RCT: 3 - and 5-year follow-up. J Clin Periodontol. 2018;45(6):744-53.

46. Tomasi C, et al. Efficacy of reconstructive surgical therapy at periimplantitis-related bone defects. A systematic review and metaanalysis. J Clin Periodontol. 2019;46(Suppl 21):340-56.

47. Schwarz F, et al. Impact of defect configuration on the clinical outcome following surgical regenerative therapy of peri-implantitis. J Clin Periodontol. 2010;37(5):449-55.
48. Schwarz F, et al. Comparison of naturally occurring and ligatureinduced peri-implantitis bone defects in humans and dogs. Clin Oral Implants Res. 2007;18(2):161-70.

49. Schwarz F, Sahm N, Becker J. Combined surgical therapy of advanced peri-implantitis lesions with concomitant soft tissue volume augmentation. A case series. Clin Oral Implants Res. 2014;25(1): $132-6$.

50. Nart J, et al. Vancomycin and tobramycin impregnated mineralized allograft for the surgical regenerative treatment of peri-implantitis: a 1-year follow-up case series. Clin Oral Investig. 2018;22(6):2199207.

51. Ramanauskaite A, et al. Clinical outcomes following surgical treatment of peri-implantitis at grafted and non-grafted implant sites: a retrospective analysis. Int J Implant Dent. 2018;4(1):27.

52. Isler SC, et al. The effects of ozone therapy as an adjunct to the surgical treatment of peri-implantitis. J Periodontal Implant Sci. 2018;48(3):136-51.

Publisher's Note Springer Nature remains neutral with regard to jurisdictional claims in published maps and institutional affiliations. 